\title{
EMOCIONES NEGATIVAS Y SALUD
}

\section{Negative emotions and health}

José Antonio Piqueras Rodríguez, Victoriano Ramos Linares Agustín Ernesto Martínez González, Luís Armando Oblitas Guadalupe*

\begin{abstract}
Resumen
Los autores llevan a cabo una revisión del estado de la cuestión en la actualidad del tema emociones negativas y salud. En primer lugar tratan el concepto de emoción y, a continuación, cuales son las principales características de las emociones negativas. Luego abordan la cuestión de la relación entre emociones negativas y salud-enfermedad, para finalizar con algunas ideas que sinteticen lo tratado a lo largo del artículo.
\end{abstract}

Palabras clave: Emociones negativas, salud

\section{Abstract}

The authors make an analysis of the present situation of the topic on negative emotions and health. First of all, they talked about the concept of emotion and then the main characteristics of negative emotions. Later they deal with the relation between negative emotions and health-sickness, and they finally end with some ideas that summarize what was dealt with in the whole article.

Key words: Negative emotions, health.

\footnotetext{
* José Antonio Piqueras Rodríguez, Ph.D. Universidad Miguel Hernández de Elche. Área de Personalidad, Evaluación y Tratamiento Psicológico, Departamento de Psicología de la Salud. E-mail: jpiqueras@umh.es
} 


\section{INTRODUCCIÓN}

Las emociones son reacciones psicofisiológicas de las personas ante situaciones relevantes desde un punto de vista adaptativo, tales como aquellas que implican peligro, amenaza, daño, pérdida, éxito, novedad, etc. Estas reacciones son de carácter universal, bastante independientes de la cultura, producen cambios en la experiencia afectiva (dimensión cognitivo-subjetiva), en la activación fisiológica (dimensión fisiológicaadaptativa) y en la conducta expresiva (dimensión conductual-expresiva). Además, desde un punto de vista psicológico emociones tales como la alegría, el miedo, la ansiedad o la ira son emociones básicas que se dan en todos los individuos de las más diversas culturas; poseen un sustrato biológico considerable; son esencialmente agradables o desagradables; nos activan y forman parte de la comunicación con los demás y; a su vez, pueden actuar como poderosos motivos de la conducta (Cano-Vindel y Miguel-Tobal, 2001).

Entre las emociones podemos distinguir al menos dos grupos: las positivas y las negativas. El miedoansiedad, la ira, la tristeza-depresión y el asco son reacciones emocionales básicas que se caracterizan por una experiencia afectiva desagradable o negativa y una alta activación fisiológica. Las tres primeras son las emociones más estudiadas en relación con el proceso salud-enfermedad y a las cuatro se las engloba en la categoría de "emociones negativas". Estas reacciones tienen una función preparatoria para que las personas puedan dar una respuesta adecuada a las demandas del ambiente, por lo que se consideran respuestas eminentemente adaptativas para el individuo. Sin embargo, en ocasiones encontramos que algunas de estas reacciones pueden transformarse en patológicas en algunos individuos, en algunas situaciones, debido a un desajuste en la frecuencia, intensidad, adecuación al contexto, etc. Cuando tal desajuste acontece y se mantiene un cierto tiempo, puede sobrevenir un trastorno de la salud, tanto mental (trastorno de ansiedad, depresión mayor, ira patológica, etc.) como física (trastornos cardiovasculares, reumatológicos, inmunológicos, etc.).

A pesar de estas afirmaciones, en las que probablemente la mayoría de profesionales de la salud estaría de acuerdo, no existe tanta coincidencia en cuanto a otras cuestiones relacionadas. Entre otras, existe cierta controversia en relación con qué entendemos por emociones negativas y positivas, cuál es la verdadera naturaleza de las relaciones entre estas emociones negativas y las diferentes condiciones de salud-enfermedad y un largo etcétera. Por ello, es preciso profundizar en el conocimiento de estas cuestiones. En las próximas páginas pretendemos llevar a cabo una revisión del estado de la cuestión en la actualidad. Primero trataremos el concepto de emoción $\mathrm{y}$, a continuación, cuales son las principales características de las emociones negativas. Luego abordaremos la cuestión de la relación entre emociones negativas y salud-enfermedad, para finalizar con algunas ideas que sinteticen lo tratado a lo largo del capítulo.

\section{CONCEPTO DE EMOCIÓN}

Las emociones básicas constituyen patrones individuales de conducta expresiva, cada uno asociado a un patrón específico de activación fisiológica y a una experiencia cognitiva-subjetiva o sentimiento específico, todo ello implicando la existencia de un substrato neuroanatómico específico para cada emoción (Piqueras, Martínez, Ramos, Rivero y García-López, 2006).

El miedo, la ira, la tristeza, la alegría, la sorpresa y el asco son las emociones básicas para las que existe un mayor consenso. Desde Darwin hasta autores más contemporáneos, entre ellos Tomkins, Izard y Ekman, se ha resaltado la importancia de las emociones en cuanto a su función adaptativa para la supervivencia del individuo y de la especie. Todos estos autores asumen la existencia de emociones básicas que son producto de la evolución filogenética y ontogenética y que están relacionadas con estados biológicamente significativos como son la procreación, la cría y la amenaza a la integridad de los individuos y de la especie.

Por tanto, podemos entender como emoción una experiencia multidimensional con al menos tres sistemas de respuesta: cognitivo/subjetivo; conductual/ expresivo y fisiológico/adaptativo. Para entender la emoción es conveniente atender a estas tres dimensiones por las que se manifiesta, teniendo en cuenta que suele aparecer cierta desincronía entre los tres sistemas de 
respuesta. Además, la notoriedad de cada una de estas dimensiones varía en función de la emoción en concreto, la persona en particular, o la situación determinada. Es más, en la mayoría de ocasiones las diferencias entre los distintos modelos teóricos de la emoción se deben únicamente al papel que otorgan a cada una estas tres dimensiones (Chóliz, 2005).

Por otra parte, han existido gran cantidad de intentos por analizar la emoción en sus componentes o dimensiones principales para facilitar tanto su clasificación, como la distinción entre las mismas (p. ej., Spencer, 1890; Wundt, 1896; Woodworth, 1938; Engen, Levy y Schlosberg, 1958; etc.). A pesar de ello, las únicas dimensiones que son aceptadas por prácticamente todos los autores son la dimensión agrado-desagrado y la intensidad de la reacción emocional (Zajonc, 1980). No obstante, atendiendo únicamente a éstas no puede establecerse una clasificación exhaustiva y excluyente de todas las reacciones afectivas, puesto que emociones como la ira o el odio pueden ser desagradables e intensas y no se trata del mismo tipo de emoción. Por tanto, la experiencia emocional, lo que pensamos y sentimos durante una reacción emocional, se suele clasificar según estos dos ejes o dimensiones fundamentales: placer-desagrado e intensidad. En otras palabras, las emociones suelen provocar sensaciones agradables o desagradables y pueden ser más o menos intensas (Chóliz, 2005). Aunque, como ya hemos dicho anteriormente, es difícil lograr una clasificación exhaustiva de todas las emociones posibles en base a dimensiones independientes, la dimensión agradodesagrado es exclusiva y característica de las emociones, de forma que todas las reacciones afectivas se comprometen en dicha dimensión en alguna medida. Esta dimensión de placer-displacer sería la característica definitoria de la emoción respecto a cualquier otro proceso psicológico. De forma similar, Oatley (1992) señala que lo realmente definitorio y diferenciador de las emociones es la disposición para la acción y la “cualidad fenomenológica". Así, una emoción podría definirse como una experiencia afectiva en cierta medida agradable o desagradable, que supone una cualidad fenomenológica característica y que compromete tres sistemas de respuesta: cognitivosubjetivo, conductual-expresivo y fisiológicoadaptativo.
Otra forma de clasificar las emociones es haciendo referencia a las funciones propias de las mismas. Parece verdaderamente relevante el hecho de que desde Darwin, pasando por James, Cannon, Selye, etc., se ha venido reconociendo que la expresión de las emociones en los animales y en el hombre cumple una función universal adaptativa, social y motivacional. En este sentido, es bien sabido que todas las emociones tienen alguna función que les confiere utilidad y permite que el sujeto ejecute con eficacia las reacciones conductuales apropiadas y ello con independencia de la cualidad hedónica que generen. Es más, incluso las emociones más desagradables tienen funciones importantes en la adaptación social y el ajuste personal. A continuación se describe brevemente cada una de estas funciones, siguiendo en parte a Chóliz (2005).

\section{Función adaptativa}

Una de las funciones más importantes de la emoción es la de preparar al organismo para ejecutar eficazmente la conducta exigida por las condiciones ambientales, movilizando la energía necesaria para ello, así como dirigiendo la conducta (acercando o alejando) hacia un objetivo determinado. Plutchik (1980) destaca ocho funciones principales de las emociones y aboga por establecer un lenguaje funcional que identifique cada una de dichas reacciones con la función adaptativa que le corresponde. La correspondencia entre la emoción y su función son las siguientes: miedoprotección; ira-destrucción; alegría-reproducción; tristeza-reintegración; confianza-afiliación; ascorechazo; anticipación-exploración; sorpresaexploración. Relacionada con esta función adaptativa se encuentra la evidencia de que las emociones están íntimamente relacionadas con diversos sistemas fisiológicos que forman parte del proceso que podríamos denominar "salud-enfermedad", así como de que las emociones juegan un papel importante en el bienestar/malestar psicológico de los individuos, que no deja de ser un indicador del grado de ajuste/ adaptación del individuo.

\section{Función social}

Las emociones también cumplen una función importante en la comunicación social. Según Izard (1993) existen varias funciones sociales de las emociones, como son: i) facilitar la interacción social; ii) controlar la conducta de los demás; iii) permitir la 
comunicación de los estados afectivos y, iv) promover la conducta prosocial. Por ejemplo, una emoción como la felicidad favorece los vínculos sociales y las relaciones interpersonales, mientras que la ira pueden generar repuestas de evitación o de confrontación. En resumen, las emociones juegan un doble papel en su función comunicativa. En primer lugar, la expresión de las emociones puede considerarse como una serie de estímulos discriminativos que facilitan la realización de las conductas apropiadas por parte de los demás. Así, en muchos casos la revelación de las experiencias emocionales es saludable y beneficiosa, tanto porque reduce el trabajo fisiológico que supone la inhibición como por el hecho de que favorece la creación de una red de apoyo social ante la persona afectada. Sin embargo, en ocasiones los efectos sobre los demás pueden llegar a ser perjudiciales, hecho éste que está constatado por la evidencia de que aquéllos que proveen apoyo social al afligido sufren con mayor frecuencia trastornos físicos y mentales. En segundo lugar, la propia represión de las emociones tiene una evidente función social, ya que en un principio se trata de un proceso claramente adaptativo, por cuanto que es socialmente necesaria la inhibición de ciertas reacciones emocionales que podrían alterar las relaciones sociales y afectar incluso a la propia estructura y funcionamiento de grupos y cualquier otro sistema de organización social.

\section{Función motivacional}

La relación entre emoción y motivación es íntima, ya que se trata de una experiencia presente en cualquier tipo de actividad que posee las dos principales características de la conducta motivada, dirección e intensidad. La emoción energiza la conducta motivada. Una conducta "cargada" emocionalmente se realiza de forma más vigorosa. Así, la emoción tiene la función adaptativa de facilitar la ejecución eficaz de la conducta necesaria en cada exigencia. Así, la ira facilita las reacciones defensivas, la alegría la atracción interpersonal, la sorpresa la atención ante estímulos novedosos, etc. Por otro lado, la emoción dirige la conducta, en el sentido que facilita el acercamiento o la evitación del objetivo de la conducta motivada en función de las características de agrado-desagrado de la emoción. La función motivacional de la emoción sería congruente con lo que hemos comentado anteriormente, de la existencia de las dos dimensiones principales de la emoción: dimensión de agrado-desagrado e intensidad de la reacción afectiva.
En resumen, si bien algunas de las principales discusiones teóricas actuales giran en torno a si existen emociones básicas y si el reconocimiento de las mismas es universal, lo cierto es que existen ciertos patrones de reacción afectiva distintivos, generalizados y que suelen mostrar una serie de características comunes en todos los seres humanos. Se trata de las emociones de alegría, tristeza, ira, sorpresa, miedo y asco. Podemos defender incluso que se caracterizan por una serie de reacciones fisiológicas o motoras propias, así como por la facilitación de determinadas conductas que pueden llegar a ser adaptativas. Por último, tradicionalmente se suelen diferenciar dos grupos de emociones en base a la dimensión de agrado-desagrado. Por una parte, nos encontramos con las emociones positivas, que se caracterizan porque generan una experiencia agradable. Ejemplos de este tipo de emociones son la alegría, la felicidad o el amor. Por otra parte, existe una serie de emociones cuya experiencia emocional es desagradable y que han sido profusamente investigadas en su relación con la salud mental y física. En el siguiente apartado vamos a repasar algunas de las características principales de las emociones negativas.

\section{EMOCIONES NEGATIVAS (MIEDO, TRISTEZA, IRA Y ASCO)}

A pesar de la pujante aparición de la psicología positiva en los últimos años, el estudio de las "emociones negativas" ha tenido durante todo el siglo $\mathrm{XX}$ y sigue teniendo mucha fuerza en el desarrollo de la investigación de la ciencia psicológica. Se refiere a las emociones que producen una experiencia emocional desagradable, como son el miedo-ansiedad, la ira y la tristeza-depresión, las tres emociones negativas más estudiadas. Nosotros también añadimos el asco, que en los últimos 15-20 años también ha sido objeto de interés por parte de la comunidad científica. Describamos brevemente cada una de estas emociones.

\section{Miedo}

El miedo y la ansiedad son las emociones que han generado mayor cantidad de investigación y sobre las que han desarrollado un arsenal de técnicas de intervención mayor desde todas las orientaciones teóricas psicológicas. La expresión patológica del miedo son los trastornos por ansiedad, que están relacionados con una respuesta de ansiedad ante un 
peligro inexistente o desproporcionada para lo que la situación requiere. Es una de las reacciones que produce mayor cantidad de trastornos mentales, conductuales, emocionales y psicosomáticos. La distinción entre fobia y miedo podría concretarse en que la reacción de miedo se produce ante un peligro real y la reacción es proporcionada a éste, mientras que en la fobia la respuesta de ansiedad es desproporcionadamente intensa (o innecesaria) con la supuesta peligrosidad del estímulo. Para nuestro fin, no distinguiremos entre ambos términos.

Siguiendo a Sandín y Chorot (1995), el miedoansiedad puede ser definida como una respuesta del organismo que se desencadena ante una situación de amenaza o peligro físico o psíquico, cuyo objeto es dotar al organismo de energía para anular o contrarrestar el peligro mediante una respuesta (conducta de huida o de agresión).

Este mecanismo funciona de forma adaptativa y pone en marcha dicho dispositivo de alerta ante estímulos o situaciones que son potencialmente agresoras o amenazantes. Existe un continuo entre la ansiedad adaptativa y la ansiedad clínica, ambas tienen la misma fenomenología: cogniciones, neurofisiología y respuesta motoras de defensa o ataque. Sabemos que una cierta cantidad de ansiedad (normal) es necesaria para realizar ciertas tareas, para resolver problemas de un modo eficaz. Existe además una relación simétrica entre nivel de ansiedad (visto como nivel de motivación) y rendimiento (desarrollo de una tarea, mecanismo de resolución de problemas). Así, la relación entre ansiedad y rendimiento viene expresada por una curva en forma de U invertida. Según la Ley de Yerkes-Dodson (1908), un déficit de ansiedad conlleva una eficacia de acción baja, mientras que una ansiedad óptima ante determinados problemas propicia el aumento, de forma deseable, de la ejecución o eficacia. Sin embargo, la ansiedad excesiva o clínica actúa interfiriendo el rendimiento en todos los ámbitos del ser humano y se torna en clínica en las siguientes situaciones:

- Cuando el estímulo presentado es inofensivo y conlleva una compleja respuesta de alerta.

- Cuando la ansiedad persiste en el tiempo, superando lo meramente adaptativo y los niveles de alerta persisten.
- Cuando los niveles de alerta y la ansiedad interrumpen el rendimiento del individuo y las relaciones sociales.

Como ya hemos dicho, la ansiedad clínica tiene varios conceptos relacionados como el miedo y las fobias. Se tratarían de reacciones equivalentes, pero en el primer caso se produciría ante una situación de amenaza real mientras que en el segundo serían ante un peligro no real o sobredimensionado. En cualquier caso a nivel operativo bastantes autores identifican dichos conceptos como sinónimos (Sandín y Chorot, 1995).

El análisis de las características principales de la ansiedad como emoción negativa viene resumido en el tabla 1. Entre éstas, especial interés tiene el conocido triple nivel de respuesta: fisiológico, cognitivo y motor.

\section{Tristeza}

Tradicionalmente como una de las emociones desagradables, aunque no siempre se puede decir que es negativa. Existe gran variabilidad cultural e incluso algunas culturas no poseen palabras para definirla. No obstante, la tristeza-depresión, al igual que cualquier otra emoción, tiene una función filogenética adaptativa para recabar la atención y el cuidado de los demás, constituir un modo de comunicación en situaciones de pérdida o separación, o ser un modo de conservar "energía" para poder hacer frente a ulteriores procesos de adaptación (Whybrow, Akiskal y McKinney, 1984). Desde el punto de vista de la relación entre las emociones negativas y la respuesta de estrés, la experiencia de estado de ánimo triste dependería de la evaluación cognitiva realizada sobre la demanda situacional y los recursos que posee el sujeto para afrontar la situación, que en tal caso sería negativa y con frecuencia predominaría cuando el estresor/es se cronifica/n (Beck et al., 1983).

Otro hecho bastante común es que la ansiedad suele aparecer asociada al cuadro depresivo. En estos casos los síntomas de tensión suelen interferir en la capacidad de disfrute del sujeto respecto a las actividades agradables y se intensifican las desagradables. En tal caso, al igual que la ansiedad en su forma clínica, estos sentimientos podrían por su duración, frecuencia e 
intensidad transformarse en una depresión clínica e interferir en la capacidad adaptativa de la persona que los padece. Todo ello resulta coherente con la idea bastante aceptada en la actualidad de que el estrés tiene un importante papel en la génesis de la depresión (Lewinsohn, Gotlib y Hautzinger, 1997).

En su forma clínica, la depresión es un trastorno del estado de ánimo caracterizado por una sensación de tristeza intensa superior a dos meses. Se produce por diversas causas: acontecimientos de la vida diaria (relacionados con pérdidas o incapacidad para hacerles frente -indefensión-), cambios químicos en el cerebro, efecto secundario de medicamentos, diversos trastornos físicos o médicos. Los síntomas de la depresión no son los mismos en todos los individuos. La mayoría de sujetos dejan de tener interés por las actividades cotidianas, sienten fatiga o sensación de lentitud, problemas de concentración, trastornos del sueño, sentimientos de culpa, inutilidad o desesperanza, aumento o pérdida de apetito o de peso, disminución del deseo sexual, ideación suicida y pensamientos negativos sobre si mismo, etc. (Beck et al., 1983; Ellis, $1981 ; 1990)$.

Por lo tanto, la depresión es el resultado final de la interacción de múltiples factores constitucionales, evolutivos, ambientales e interpersonales, que modifican las pautas de neurotransmisión entre los hemisferios cerebrales y el sistema límbico. En la tabla 2 se muestran las características principales de esta emoción.

\section{Ira}

La ira es considerada una emoción negativa por la mayoría de los teóricos de la emoción. Esta emoción puede ser concebida atendiendo a tres tipos de definiciones de la valencia emocional (Lazarus, 1991), es decir, las emociones se pueden entender como positivas o negativas en base a: (i) las condiciones que evocan la emoción; (ii) las consecuencias adaptativas de la emoción o; (iii) la experiencia subjetiva de la emoción. Así, en primer lugar, la ira puede verse como negativa debido a las condiciones que evocan la emoción, ya que suele ser evocada por acontecimientos aversivos. En segundo lugar, la ira se puede calificar como positiva o negativa cuando es entendida desde el punto de vista de sus consecuencias adaptativas, dependiendo del resultado de una situación particular. Finalmente, la ira se podría ver como positiva o negativa en función de la sensación subjetiva o evaluación de la emoción, dependiendo de si un individuo siente placer/ gusto o displacer/aversión tras la experiencia subjetiva de ira. Algunos científicos se centran en las condiciones del estímulo al definir la emoción (p. ej., una ofensa percibida causa ira), mientras que otros se centran en las respuestas evocadas cuando definen la emoción (p. ej., la ira implica ciertos cambios fisiológicos, expresiones conductuales y sensaciones subjetivas). Las definiciones basadas en el estímulo indican que la evaluación del individuo del estímulo que causa la emoción determina la valencia de la emoción (Lazarus, 1991). Con estas definiciones, la relación beneficiosa o dañina persona-ambiente se considera la manera más importante y más frecuente de distinguir las emociones positivas de las negativas. Así, según la definición basada en el estímulo, la ira es una emoción negativa. Las definiciones basadas en la respuesta indican que la evaluación subjetiva del individuo de los sentimientos determina la valencia. Respecto a otras características típicas de la ira la tabla 3 muestra un resumen.

\section{Asco}

El asco es una de las reacciones emocionales en las que las sensaciones fisiológicas son más patentes. La mayoría de las reacciones de asco se generan por condicionamiento interoceptivo. Está relacionado con trastornos del comportamiento, tales como la anorexia y bulimia, pero puede ser el componente terapéutico principal de los tratamientos basados en condicionamiento aversivo, tales como la técnica de fumar rápido, así como en el tratamiento para reducir las náuseas y vómitos anticipatorios a la quimioterapia (Chóliz, 2005).

Esta reacción emocional suele darse ante estímulos desagradables (químicos fundamentalmente) que son potencialmente peligrosos o molestos. Suele ser muy proclive a los condicionamientos aversivos, siendo los Estímulos Condicionados mayoritariamente olfativos o gustativos.

La actividad fisiológica típica de esta emoción suele ser el aumento en la reactividad gastrointestinal y la tensión muscular. No obstante, las dos reacciones fisiológicas más características y distintivas de esta 
emoción son las sensaciones de náusea y el aumento de la salivación. Por otra parte, a diferencia de emociones como el miedo y la ira, donde predomina la respuesta simpática del sistema nervioso autónomo, en el asco se ha contrastado una mayor predominancia de la respuesta parasimpática (Rozin, Haidt y McCauley, 2000).

Entre las funciones reconocidas se encuentran la generación de respuestas de escape o evitación de situaciones desagradables o potencialmente dañinas para la salud. Aunque los estímulos incondicionados suelen estar relacionados con la ingesta de forma que la cualidad fundamental es olfativa u olorosa, los estímulos condicionados pueden asociarse a cualquier otra modalidad perceptiva (escenas visuales, sonidos, etc.). Además también parece demostrado que no se puede restringir la emoción de asco a estímulos relacionados con alimentos en mal estado o potencialmente peligrosos para la salud, ya que lo cierto es que esta reacción emocional también se produce ante cualquier otro tipo de estimulación que no tenga por qué estar relacionada con problemas gastrointestinales. Es más, incluso puede producirse reacción de asco ante alimentos nutritivos y en buen estado. Otra de las funciones reconocidas es la de potenciar hábitos saludables, higiénicos y adaptativos. Por último, tiene un papel motivador fundamental de cara a energizar conductas que implican la necesidad de evitación o alejamiento del estímulo, siendo características las sensaciones desagradables, tales como la náusea, cuando el estímulo es oloroso o gustativo (véase tabla 4).

Tabla 1. Características del miedo (tomado parcialmente de Chóliz, 2005).

\begin{tabular}{|c|c|}
\hline Instigadores & $\begin{array}{l}\text { - Situaciones potencialmente peligrosas o Estímulos Condicionados que producen Respuestas Condicionadas de } \\
\text { miedo. Los EC's pueden ser variados y carecer objetivamente de peligro. } \\
\text { - Situaciones novedosas y misteriosas, especialmente en niños } \\
\text { - Procesos de valoración secundaria que interpretan una situación como peligrosa. } \\
\text { - Dolor y anticipación del dolor. } \\
\text { - Pérdida de sustento y, en general, cambio repentino de estimulación. }\end{array}$ \\
\hline $\begin{array}{l}\text { Activ } \\
\text { fisiol }\end{array}$ & $\begin{array}{l}\text { - Sistema Nervioso Autónomo (División simpática adrenal): activación rápida al identificar cualquier amenaza de un } \\
\text { modo automático e involuntario. Elevación de la frecuencia cardiaca (taquicardia), de la presión arterial, dilatación de } \\
\text { los bronquios, aumento de la tensión muscular, incremento de la conductancia y de las fluctuaciones de la misma. } \\
\text { - Sistema Neuroendocrino (hipotálamo-hipófiso-suprarrenal): Activación más lenta, de efectos más duraderos que } \\
\text { SNA y que requiere una exposición más prolongada a amenaza. }\end{array}$ \\
\hline & $\begin{array}{l}\text { - Lazarus y Faulkman (1986): valoración primaria de amenaza y valoración secundaria de ausencia de estrategias de } \\
\text { afrontamiento apropiadas. }\end{array}$ \\
\hline $\begin{array}{l}\text { Procesos } \\
\text { cognitivos }\end{array}$ & $\begin{array}{l}\text { - Reducción de la eficacia de los procesos cognitivos, obnubilación. Focalización de la percepción en el estímulo } \\
\text { temido. }\end{array}$ \\
\hline im & $\begin{array}{l}\text { - Pensamientos negativos automáticos (Beck et al., 1983) y creencias irracionales (Ellis, 1981, 1990) antes y durante la } \\
\text { situación, interpretándola como peligrosa o bien valorando la propia incapacidad para afrontarla. } \\
\text { - Preocupación, anticipación, hipervigilancia, temor, inseguridad, sensación de pérdida de control y la percepción de } \\
\text { fuertes cambios fisiológicos, etc. }\end{array}$ \\
\hline Función & $\begin{array}{l}\text { - Facilitación de respuestas de escape o evitación de la situación peligrosa: procedimientos de reforzamiento negativo. } \\
\text { - Al prestar una atención casi exclusiva al estímulo temido, facilita que el organismo reaccione rápidamente ante el } \\
\text { mismo. } \\
\text { - Moviliza gran cantidad de energía para ejecutar las respuestas de manera mucho más intensa que en condiciones } \\
\text { normales. Si la reacción es excesiva, la eficacia disminuye, según la relación entre activación y rendimiento de Yerkes } \\
\text { y Dodson (1908). }\end{array}$ \\
\hline $\begin{array}{l}\text { Experiencia } \\
\text { subjetiva }\end{array}$ & $\begin{array}{l}\text { - Se trata de una de las emociones más intensas y desagradables. Genera aprensión, desasosiego y malestar. } \\
\text { - Preocupación, recelo por la propia seguridad o por la salud. } \\
\text { - Sensación de pérdida de control. }\end{array}$ \\
\hline $\begin{array}{l}\text { Expresión } \\
\text { conductual }\end{array}$ & $\begin{array}{l}\text { - Confrontación (acciones dirigidas hacia la acción como el contraataque, la reacción agresiva, es decir, la ira); } \\
\text { distanciamiento (evitaciones, escapes); autocontrol; búsqueda de apoyo social, etc. } \\
\text { - Objetivo de las conductas: mitigar o aliviar el malestar físico o psicológico generado (evitación, escape) que pueden } \\
\text { derivar en conductas no saludables tales como beber, fumar, llevar una mala dieta, etc. }\end{array}$ \\
\hline
\end{tabular}


Tabla 2. Características de la tristeza (tomado parcialmente de Chóliz, 2005).

Instigadores

Actividad fisiológica

Procesos cognitivos implicados

Función

Experiencia subjetiva

Expresión conductual
- Separación física o psicológica, pérdida o fracaso; decepción, especialmente si se han desvanecido esperanzas puestas en algo; situaciones de indefensión, ausencia de predicción y control; ausencia de actividades reforzadas y conductas adaptativas; dolor crónico.

- SNC: disfunción central de la neurotransmisión (descompensación de las monoaminas neurotransmisoras noradrenalina y serotonina, y posiblemente, también de la acetilcolina y de las endorfinas).

- Sistema Neuroendocrino (hipotálamo-hipófiso-suprarrenal): Actividad fisiológica elevada y sostenida con ligero aumento en frecuencia cardiaca, presión sanguínea y resistencia eléctrica de la piel (perfil bioquímico similar al propio de situaciones de estrés: activación eje hipotalámico-hipofisiario-córticosuprarrenal; perturbación de los ritmos circadianos de cortisol, etc.

- Valoración de pérdida o daño que no puede ser reparado.

- Focalización de la atención en las consecuencias a nivel interno de la situación.

- Proceso cognitivo característico de la depresión (tríada cognitiva, esquemas depresivos y errores en el procesamiento de la información (Teoría de Beck; Beck et al., 1983).

- Teoría de la Desesperanza (Abramson et al., 1978) y Teoría de la Indefensión Aprendida (Seligman, 1981).

- Cohesión con otras personas, especialmente con aquéllos que se encuentran en la misma situación.

- Disminución en el ritmo de actividad. Valoración de otros aspectos de la vida que antes no se les prestaba atención.

- Comunicación a los demás que no se encuentra bien y ello puede generar ayuda de otras personas, así como apaciguamiento de reacciones de agresión por parte de los demás, empatía, o comportamientos altruistas.

- Desánimo, melancolía, desaliento; pérdida de energía.

- Reducción generalizada en la frecuencia de las conductas y pérdida de reforzadores positivos contingentes a la conducta.

- Pérdida en la efectividad de tales reforzadores (o la incapacidad de hacer frente a una situación -indefensión aprendida).

- Pérdidas/incapacidad debidas a factores ambientales (rupturas de pareja) o intrapersonales (déficit habilidades sociales).

- Evidencia respecto antecedentes de sucesión de eventos vitales estresantes o una historia previa de adversidades y estrés psicosocial (Kessler, 1997).

Tabla 3. Características de la ira (tomado parcialmente de Chóliz, 2005).

\footnotetext{
- Estimulación aversiva, tanto física o sensorial, como cognitiva. $\begin{array}{ll}\text { Instigadores } & \text { Condiciones que generan frustración, interrupción de una conducta motivada, situaciones injustas, o atentados contra } \\ \text { valores morales. }\end{array}$

- Extinción de la operante, especialmente en programas de reforzamiento continuo.

- Inmovilidad, restricción física o psicológica.

Actividad - Elevada actividad neuronal y muscular.

fisiológica - Reactividad cardiovascular intensa (elevación en los índices de frecuencia cardiaca, presión sistólica y diastólica).

Procesos

cognitivos

implicados

- Focalización de la atención en los obstáculos externos que impiden la consecución del objetivo o son responsables de la frustración.

- Obnubilación, incapacidad o dificultad para la ejecución eficaz de procesos cognitivos.

Función

- Movilización de energía para las reacciones de autodefensa o de ataque.

no siempre concluye en agresión, al menos sirve para inhibir las reacciones indeseables de otros sujetos e incluso evitar una situación de confrontación.

Experiencia - Sensación de energía e impulsividad, necesidad de actuar de forma intensa e inmediata (física o verbalmente) para subjetiva solucionar de forma activa la situación problemática.

- Se experimenta como una experiencia aversiva, desagradable e intensa. Relacionada con impaciencia.

- En general aproximación al estímulo, objeto o situación.

- Tres estilos distintos de expresión de la ira:

Expresión conductual

- Supresión de la ira (anger-in). Afrontamiento de la situación reprimiendo la expresión verbal o física, aunque persista activación alta.

- Expresión de la ira (anger-out). Afrontamiento de la situación manifestando conductas airadas verbal o físicamente, hacia otras personas u objetos, aunque sin intención de producir daño.

- Control de la ira (anger-control). Afrontamiento de la situación canalizando la energía emocional proyectándola hacia fines constructivos, tales maximizar la posible resolución positiva del conflicto.
} 
Tabla 4. Características del asco (tomado parcialmente de Chóliz, 2005).
Instigadores
- Estímulos desagradables (químicos fundamentalmente) potencialmente peligrosos o molestos.
- EC's condicionados aversivamente. Los EI's suelen ser olfativos o gustativos.
Actividad
fisiológica
- Aumento en reactividad gastrointestinal.
- Tensión muscular
- Náusea e incremento de la salivación.
- Mayor predominancia de la división parasimpática del sistema nervioso autónomo frente a otras emociones como el miedo y la ira.
Procesos
cognitivos
implicados
- Experiencia subjetiva de repulsa, de corta duración.
- Puede provocar reacciones de humor en determinados contextos que elicitan sensaciones de asco.
- Generación de respuestas de escape o evitación de situaciones desagradables o potencialmente dañinas para la salud. Los estímulos suelen estar relacionados con la ingesta de forma que la cualidad fundamental es olfativa u olorosa, si bien los EC's pueden asociarse a cualquier otra modalidad perceptiva (escenas visuales, sonidos, etc.)
Función
- A pesar de que algunos autores restringen la emoción de asco a estímulos relacionados con alimentos en mal estado o potencialmente peligroso para la salud, lo cierto es que esta reacción emocional también se produce ante cualquier otro tipo de estimulación que no tenga por qué estar relacionada con problemas gastrointestinales. Incluso puede producirse reacción de asco ante alimentos nutritivos y en buen estado.
- Potenciar hábitos saludables, higiénicos y adaptativos

Experiencia - Necesidad de evitación o alejamiento del estímulo. Si el estímulo es oloroso o gustativo aparecen sensaciones subjetiva gastrointestinales desagradables, tales como náusea.

Expresión

- Expresión facial: retracción del labio superior de la boca y arrugamiento de la nariz.

conductual

\footnotetext{
- Distanciamiento del objeto, evento o situación.

- Rechazo.
}

\section{EMOCIONES NEGATIVAS Y PROCESO SALUD-ENFERMEDAD.}

Hasta aquí se ha hablado de las emociones como reacciones básicas adaptativas, pero tanto la ansiedad y la depresión como también la ira y el asco (si bien ninguna de ellas tiene su propia categoría diagnóstica en los manuales psiquiátricos, aun siendo condiciones con claras implicaciones clínicas) pueden dar lugar a manifestaciones clínicas de estas emociones, donde el carácter adaptativo de las mismas se pierde y se convierte en un problema que interfiere y genera malestar clínicamente significativo. Estas formas se corresponderían aproximadamente con los trastornos de ansiedad y afectivos del Eje I del DSM y de la CIE10 , cuando nos referimos a los trastornos psiquiátricos, así como también a algunos de los factores psicológicos propuestos por las más recientes ediciones DSM bajo la denominación de "Factores psicológicos que afectan a la condición médica" (APA, 2000) o con la categoría "Factores psicológicos y del comportamiento en trastornos o enfermedades clasificadas en otro lugar" en el CIE-10 (OMS, 1992), cuando nos referimos a las manifestaciones emocionales desadaptativas asociadas a las enfermedades físicas.

En resumen, parece que podemos afirmar que las emociones influyen sobre la salud-enfermedad a través de los diferentes trastornos clínicos así como a través de sus propiedades motivacionales para modificar las conductas "saludables" (ejercicio físico moderado, dieta equilibrada, descanso, ocio, etc.) y "no saludables" (abuso de alcohol, tabaco, sedentarismo) (Chóliz, 2005). Pero no solo la ansiedad, la depresión, la "ira patológica" o el "asco patológico" pueden afectar adversamente a una condición médica, sino que la evidencia empírica nos muestra que bien de forma específica bien asociadas a enfermedades físicas existe una alta prevalencia de problemas de ansiedad, depresión e ira que evidentemente han de ser tenidas en cuenta (Martín, 2005).

En primer lugar atenderemos a la relación entre emociones negativas y salud mental, para posteriormente abordar la relación de las primeras con 
la salud física, aunque somos conscientes que esta diferenciación obedece a un intento por facilitar las descripciones más que a una separación real entre los aspectos de salud mental y física.

\section{EMOCIONES NEGATIVAS Y SALUD MENTAL}

\section{Ansiedad}

Cuando la frecuencia, intensidad o duración de la ansiedad como respuesta emocional es excesiva puede dar lugar a la aparición de limitaciones, más o menos importantes, en la vida del individuo y en su capacidad de adaptación al medio circundante. En estos casos hablamos de ansiedad patológica o de un trastorno de ansiedad.

La presencia de fuertes reacciones o estados de ansiedad no solamente va a estar en la base de los denominados trastornos de ansiedad, sino que va a estar asociada frecuentemente a la depresión, y en general a los distintos trastornos considerados tradicionalmente como neuróticos, a buena parte de los trastornos psicóticos y a una amplia variedad de trastornos psicofisiológicos (APA, 2000).

Como puede observarse por la amplia gama de problemas relacionados con la ansiedad, podríamos afirmar que esta va a ser un elemento central en psicopatología y en buena parte de otros problemas relacionados con la salud, dando lugar a un considerable coste emocional, pero también económico para cualquier sistema sanitario.

Los trastornos de ansiedad presentan la mayor prevalencia entre los diferentes trastornos mentales, concretamente un $16,4 \%$ de la población de Norteamérica sufre algún trastorno de ansiedad al cabo de un año. (Barlow, 2002). La cronicidad de su curso se halla por delante de la de los trastornos del estado de ánimo y de las adicciones. Por lo que se refiere a España, nuestro país, la cifra de personas que en el último año han tenido algún trastorno de ansiedad asciende a dos millones cuatrocientas mil personas $(5,9 \%$; CanoVindel, 2004).

Para una revisión de los distintos trastornos de ansiedad recomendamos al lector acudir al DSM-IVTR (APA, 2000) o al CIE-10 (OMS, 1992). No obs- tante, al margen de las categorías diagnósticas incluidas en estos manuales, la ansiedad como síntoma aparece relacionada con la mayoría de trastornos afectivos, psicóticos, etc.

\section{Depresión}

La depresión es un trastorno del estado de ánimo o trastorno afectivo. El estado de ánimo deprimido es una de las condiciones psicopatológicas más frecuentes de los seres humanos (Vázquez, 1990). El estado de ánimo puede entenderse como la tendencia básica del ser humano para aportar a los estados psíquicos un tono agradable o desagradable (Villagrán, 1996), o como el estado emocional subjetivo del individuo (Friedman y Thase, 1995). Evidentemente, en la depresión, el estado de ánimo estaría asociado al polo desagradable, a la tendencia a la negatividad. La depresión, por tanto, debe entenderse como un trastorno del estado de ánimo, que se manifiesta mediante un conjunto de síntomas característicos. Una de las características principales es la bajada del estado de ánimo, aunque el estado de ánimo deprimido no debe ser el único criterio a tener en cuenta para diagnosticar depresión. "Esta distinción es muy importante porque la depresión-síntoma está presente en la mayor parte de los cuadros psicopatológicos" (Vázquez, 1990, pág. 902).

La depresión como trastorno clínico es descrita comúnmente por las sensaciones de tristeza, desesperanza, vacío, pérdida de interés y placer en actividades cotidianas durante más de 2 semanas. (APA, 2000).

Aunque es un trastorno clínico, todas las personas experimentan sentimientos transitorios disfóricos de tristeza, desesperanza, vacío, pérdida de interés o placer en las actividades cotidianas. Por tanto, aunque el término depresión se utiliza como sinónimo de tristeza, ambos conceptos no son lo mismo. Así, aunque en numerosas ocasiones se experimenta tristeza con marcado carácter negativo en situaciones cotidianas concretas, esta experiencia no se refiere a un estado de ánimo anormal o patológico sino a la reacción emocional básica de la que hemos hablado más arriba.

\section{Ira}

La ira es un estado de malestar que puede ir desde una intensidad equivalente a la irritación suave hasta 
la denominada cólera intensa. Se da en respuesta a un mal percibido que amenaza el bienestar de uno mismo o de los seres significativos o con los que el individuo se identifica. No obstante, existe un gran acuerdo acerca de la variabilidad intra e interindividual en el nivel de intensidad emocional y activación fisiológica que se experimenta al enfadarse (Nicholson et al., 2007).

La ira es una asignatura pendiente del campo de la salud mental. Aunque está ampliamente reconocida como problema de salud mental significativo, la ira persistente todavía no está reconocida como una categoría diagnóstica en ninguna clasificación psiquiátrica oficial (véase DSM-IV-TR, 2000 o CIE10, 1992). En cambio, la ansiedad y la depresión, las otras emociones negativas cuentan con numerosas categorías y especificidades tanto en el DSM-IV-TR como en el CIE-10. Además, la ira persistente tiene ramificaciones psicológicas, conductuales e incluso médicas significativas, ya que causa sufrimiento emocional significativo tanto en la persona que experimenta la emoción como en las personas implicadas. También puede representar un peligro desde el punto de vista de la seguridad pública, ya que puede conducir a la violencia doméstica u otras formas de violencia (Eckhardt, Jamison, y Vatios, 2002; Lundeberg, Stith, Penn, y Sala, 2004; Norlander y Eckhardt, 2005).

Finalmente, la ira a menudo es un componente de otros trastornos emocionales, tales como los trastornos de ansiedad y los afectivos. Es más, en muchas ocasiones el tratamiento exitoso de estas condiciones pasa por conseguir la disminución o el control de la ira persistente (Suls y Bunde, 2005). Desafortunadamente, como fenómeno clínico, la ira persistente no es tan bien conocida como otros constructos psiquiátricos. La ausencia de una categoría diagnóstica oficial en el eje I para un "trastorno de ira" por sí mismo ha obstaculizado indudablemente el progreso en este área, haciéndolo difícil llegar a estimaciones fiables de los índices de prevalencia de este problema. La evidencia empírica disponible, aunque limitada, sugiere que es legítima la existencia de un trastorno de ira por si mismo, sin la necesidad de otros diagnósticos psiquiátricas asociados, y que los problemas de ira están frecuentemente asociados con otros problemas del comportamiento o problemas sociales tales como dificultades en escuela o en el trabajo, uso de alcohol y otras drogas, dificultades financieras, dificultades legales y baja autoestima (Gorenstein et al., 2007). Al mismo tiempo, la evidencia indica que la ira se puede asociar también a trastornos psiquiátricos específicos (Suls y Bunde, 2005). Los estudios psiquiátricos de comorbilidad encuentran cierto solapamiento entre trastornos de ansiedad, trastornos depresivos y problemas de ira: un paciente que experimenta cualquiera de estas alteraciones tiene un riesgo aumentado para experimentar los otros (Gorenstein et al., 2007). En resumen, aunque la ira persistente no está reconocida en el DSM-IV-TR como un trastorno psiquiátrico, es indudablemente un problema clínico significativo. La ira persistente causa malestar emocional, se solapa con la ansiedad y la depresión, puede conducir a la violencia y es un factor de riesgo para diversos trastornos médicos como es la enfermedad cardiaca (Smith, 1992). Pero este último aspecto lo veremos en un apartado posterior.

\section{Asco}

El asco no es, ni mucho menos, una emoción que haya sido estudiada con la dedicación que lo ha sido la ansiedad, la tristeza o la ira. Sin embargo, ya existe un cierto corpus científico en relación con las asociaciones entre esta emoción otro tiempo denostada y los trastornos mentales.

Los primeros trabajos señalaron la existencia de abundantes vínculos entre el asco y la psicopatología general, especialmente con la ansiedad. Rozin et al. (2000) recogen en su capítulo de libro una síntesis de los estudios que han encontrado relaciones significativas entre ambos aspectos. Así, algunos estudios sobre diferencias individuales en sensibilidad al asco hallaron que ésta era mayor en pacientes psiquiátricos que en la población general, así como que se relacionaba con puntuaciones mayores en varias medidas de psicopatología (incluyendo neuroticismo y obsesividad). Otros estudios han informado que entre los universitarios la Escala de Asco de Haidt (1994) estaba positivamente correlacionada con la personalidad tipo obsesivo-compulsiva y con la personalidad tipo dependiente. También se han hallado relaciones conceptuales entre las obsesiones y compulsiones de limpieza y la sensibilidad al asco y al contagio. Otras investigaciones recientes indican que 
hay un déficit en la detección de expresiones faciales de asco entre los pacientes con trastorno obsesivocompulsivo. Por su parte, se ha hallado que las fobias que implican animales depredadores (tiburones o leones) provocan miedo, mientras que las fobias que implican animales que no amenazan con un daño físico significativo (ratones, arañas, serpientes, cucarachas, gusanos, etc.) están motivadas fundamentalmente por el asco. También se ha encontrado un nexo entre el asco y las fobias a la sangre/inyecciones/heridas, así como entre el asco y la depresión y entre el asco y los trastornos de la alimentación (véase Rozin et al., 2000 o Tolin, Woods y Abrazmowitz, 2006).

En resumen, en los últimos años, la investigación sobre los trastornos de ansiedad se ha centrado cada vez más en el papel potencial del asco como variable explicativa en los modelos de ansiedad (Tolin, Woods y Abramowitz, 2006). La función evolutiva que se le presume es la de prevenir de la contaminación y de la enfermedad (Izard, 1993), por lo que los modelos de los trastornos de ansiedad se han desarrollado alrededor de esta función. En este sentido, según Tolin et al. (2006), la evitación de la enfermedad parece estar implicada en las fobias específicas a animales pequeños y en las fobias a la sangre, inyecciones y heridas. Además, el asco, como muchas otras emociones, puede ser conceptualizado como estado y como rasgo. La exposición a los estímulos fóbicos puede evocar sensaciones de asco (Sawchuk, Lohr, Westendorf, Meunier y Tolin, 2002); los individuos con fobias también demuestran un predisposición general al asco (llamada sensibilidad al asco) (Sawchuk y otros., 2000) la cuál puede servir como factor subyacente de la vulnerabilidad en el desarrollo y/o el mantenimiento de las aversiones fóbicas (Tolin et al., 2007).

Para finalizar, nos gustaría hacer una aportación referente al asco como emoción. Si en el miedo la conducta de evitación o el escape representan la adaptabilidad del individuo a una situación concreta, el asco podría ser considerado como una variante, el sujeto no huiría del peligro sino que el asco en este caso actuaría como repulsivo para expulsar o alejar el peligro del organismo.

Emociones negativas y salud física.

En la actualidad existe consenso acerca de que los factores psicológicos pueden desempeñar un papel esencial en la historia natural de las enfermedades médicas. Factores como las conductas relacionadas con la salud, nuestros estados psicológicos o emocionales, las características personales y los modos de afrontamiento han demostrado ser de especial relevancia en este sentido (Oblitas, 2004). Esta afirmación es coherente con la evidencia científica actual que indica que cualquier trastorno denominado físico u orgánico suele implicar igualmente, y a distintos niveles, alteraciones psicológicas, y viceversa (Oblitas y Becoña, 2000). Entre estos factores psicológicos, se encuentran el miedo, la tristeza o la ira como reacciones emocionales negativas y sus diferentes formas clínicas, tales como los denominados trastornos emocionales o psíquicos: la ansiedad, la depresión y la ira persistente.

En este sentido, tradicionalmente las teorías del estrés psicosocial se han centrado más en las emociones negativas tales como la ira, el temor, la ansiedad, la vergüenza, la culpabilidad, la tristeza, la envidia, los celos y el asco, que en las positivas. Hoy en día hay datos suficientes para afirmar que las emociones negativas tienden a disminuir la salud. Así, algunos autores sostienen que las emociones positivas potencian la salud, mientras que las emociones negativas tienden a disminuirla (Fernández-Abascal y Palmero, 1999). Por ejemplo, en periodos de estrés en los que tenemos que responder a unas altas demandas ambientales, desarrollamos muchas reacciones emocionales negativas $\mathrm{y}$, cuando nos encontramos bajo la influencia de estos estados emocionales negativos, es más probable desarrollar ciertas enfermedades relacionadas con el sistema inmune, o adquirir determinados hábitos poco saludables, que a la larga pueden minar la salud (Becoña, Vázquez y Oblitas, 2004; Ramos, Rivero, Piqueras y García-López, 2006). En cambio, las emociones positivas, como la alegría, ayudan a mantener e incluso recuperar la salud (Nezu, Nezu y Blissett, 1988).

Las emociones negativas que más se han estudiado en cuanto a sus relaciones con trastornos de salud son la ansiedad, la depresión y la ira. Una de las reacciones emocionales que más se ha estudiado es sin duda la ansiedad como estado emocional asociado a múltiples trastornos, especialmente los trastornos de ansiedad y los trastornos psicofisiológicos. Una segunda emoción negativa que bastante estudiada es la ira, por su relación 
con los trastornos cardiovasculares (Miguel-Tobal, Casado, Cano-Vindel y Spielberger, 1997). La tristezadepresión, como emoción básica, se considera que es precursora de la depresión como patología, la cual cursa por lo general con niveles altos de ansiedad. Por último, el asco es una emoción muy poco estudiada en este sentido, pero ya existen algunas evidencias de su implicación en los procesos salud-enfermedad.

Por tanto, estamos preparados para desarrollar reacciones emocionales intensas, e incluso para repetirlas cuando sea necesario, sin que ello suponga un problema, pero a medio plazo, se pueden agotar nuestros recursos (por ejemplo, agotamiento por estrés), o alterarse algunas funciones asociadas a las reacciones emocionales, o en definitiva surgir algún problema asociado a las emociones. Lejos de la opinión mantenida por la investigación psicosomática en sus inicios respecto al carácter causal de las emociones en las enfermedades, en la actualidad se admite que los factores psicológicos pueden ser causa necesaria pero no suficiente para la aparición de determinados trastornos. Se señala la multicausalidad y la interrelación entre los diferentes factores causales como la explicación etiológica más plausible. Esta multicausalidad, considera simultáneamente los factores genéticos, ambientales, psicofisiológicos y principalmente el peso de la interacción como elemento de predisposición del individuo a padecer una determinada enfermedad, es el gran merito de la investigación psicológica actual en este campo (Kop et al., 2002).

Se han hipotetizado diversas vías para explicar las interrelaciones de los factores emocionales en el proceso de salud-enfermedad (Cano-Vindel y Tobal, 2001):

1. Las reacciones desadaptativas de ansiedad, de tristeza-depresión y de ira que alcanzan niveles demasiado intensos, o frecuentes, cuando se mantienen en el tiempo, tienden a producir cambios en la conducta, de manera que se incrementa la probabilidad de que el sujeto adopte conductas perjudiciales para la salud o que ponen en peligro nuestra salud (como las adicciones, la realización de conductas de riesgo, etc.) y de que olvide los hábitos saludables (ejercicio físico, etc.). Por ejemplo, existe relación positiva entre ansiedad y consumo de tabaco, así como entre tabaquismo y cáncer. En definitiva, estos estados y necesidades emo- cionales concretos pueden desempeñar un papel primordial en las prácticas de salud, como por ejemplo, el malestar emocional no ayuda a que la gente se implique en la realización de hábitos que favorezcan su salud tales como el no fumar, hacer ejercicio, desayunar, etc. (Leventhal, Prochaska y Hirschman, 1985).

2. Estas reacciones emocionales mantienen niveles de activación fisiológica intensos, que podrían deteriorar nuestra salud si se cronifican. Las disfunciones fisiológicas consecuentes serían arritmias, aumento crónico de la presión arterial, dispepsias, dermatitis, dolor muscular, etc. Así, los pacientes con arritmias, hipertensión esencial, cefaleas crónicas, o diferentes tipos de dermatitis, presentan niveles más altos de ansiedad, ira y activación fisiológica que la población general. En el nivel fisiológico, la evaluación de respuestas del organismo (como la tasa cardiaca) y el estudio de su relación con variables psicológicas ha dado lugar a una disciplina llamada psicofisiología, que arranca desde finales del siglo pasado. A su vez el estudio de la patología de los sistemas fisiológicos que se activan en la emoción se inicia en los años 50. Se supone que los trastornos psicosomáticos o psicofisiológicos (como algunos dolores de cabeza, o de espalda, algunas arritmias, los tipos de hipertensión arterial más frecuentes, algunas molestias gástricas, etc.) podrían estar producidos por un exceso en la intensidad y frecuencia de la activación de las respuestas fisiológicas del sistema que sufre la lesión o disfunción (cardiovascular, respiratorio, etc.). Se trataría de una disfunción de un sistema orgánico que está trabajando en exceso y mantiene esta actividad demasiado tiempo. A su vez, el trastorno produce más ansiedad y, por lo tanto, un aumento de la actividad de ese sistema, aumentando así la probabilidad de desarrollar y mantener un mayor grado de disfunción orgánica

3. Esta alta activación fisiológica puede estar asociada a un cierto grado de inmunodepresión, lo que nos vuelve más vulnerables al desarrollo de enfermedades infecciosas o de tipo inmunológico, o bien la supresión o control de estas emociones puede acarrear altos niveles de activación fisiológica y un cierto grado de inmunosupresión.

En resumen, el sufrimiento de estados emocionales negativos persistentes puede afectar al funciona- 
miento del sistema inmunológico, el sistema endocrino-metabólico y en general, de todas las funciones fisiológicas (Martín, 2005; Ramos et al., 2006).

A continuación señalaremos los trastornos sobre los que existe una mayor evidencia de la influencia de las emociones negativas. Como se observará, la práctica totalidad de sistemas orgánicos aparecen relacionados con factores psicológicos que juegan un papel importante en el inicio, desarrollo y mantenimiento de enfermedades.

\section{Cáncer}

Los enfermos oncológicos sufren reacciones psicológicas negativas ante la enfermedad de cáncer, tales como ansiedad, depresión, ira e incluso asco "patológico". Así, el impacto del diagnóstico producirá diferentes reacciones psicológicas en el paciente en función de cómo es percibido. Por ejemplo, si para el afectado el diagnóstico significa amenaza para la propia vida reaccionará con ansiedad; si representa pérdida de control sobre sí experimentará depresión y si lo percibe como una agresión y una injusticia reaccionará con rabia. Además los tratamientos mediante agentes quimioterapéuticos producen náuseas y vómitos no solo por afectación neurológica, sino también horas antes del tratamiento o ante situaciones, objetos o lugares que les recuerdan la situación, todo lo cual tiene bastante que ver con las sensaciones de asco. Se trata de un condicionamiento clásico a los efectos asociados al tratamiento químico que el enfermo generaliza. Efectivamente, cuando se acerca el día del tratamiento o recibe ciertos estímulos (situacionales, visuales, olfatorios, etc.) se desencadenan estas conductas de manera irresistible. Su prevalencia oscila, según los estudios, entre el 18\%-65\%. En el 65\% de los enfermos las náuseas y vómitos anticipatorios persisten a los 6 meses. Pero también la relación entre el asco y el cáncer ha sido investigada en otros dos sentidos. Por una parte, se ha postulado el papel del asco en el diagnóstico precoz del cáncer de páncreas y del cáncer de pulmón (Gullo et al., 2001). Por otra parte, se ha investigado hasta que punto el origen de la fobia a la sangre/inyecciones, que tiene claros vínculos con el asco, se explica por la experiencia traumática con la quimioterapia o bien con otras variables (Carey y Harris, 2005).
Estas manifestaciones psicológicas se encuentran asociadas a la gravedad de la neoplasia e influyen claramente en la respuesta a la enfermedad y en las expectativas de vida (Alonso, 2006). Así, existen varias hipótesis que intentan explicar la influencia de los factores psicológicos en el comienzo y la progresión del cáncer, que van desde su influencia en la función inmunitaria, pasando por su influencia sobre hábitos como el tabaco y el alcohol, hasta el peso que estos factores psíquicos ejercen sobre el paciente para decidir el momento de solicitar atención médica (Miralles et al., 2003).

Por ejemplo, se ha estudiado el grado de expresividad/represión emocional del enfermo oncológico, así como el posible efecto de estas variables sobre su pronóstico. En este sentido se ha descrito el patrón conductual de tipo $\mathrm{C}$ (en contraste con el tipo A descrito en Cardiología) que caracteriza al paciente cooperador y no autoritario, que suprime sus emociones negativas, particularmente la ira, y que acepta y sigue las instrucciones de las autoridades externas. Sin embargo, no se han obtenido resultados homogéneos. En el momento actual debemos concluir que si las situaciones estresantes $u$ otros factores psicológicos ejercen un efecto sobre la incidencia del cáncer, este efecto es de escasa cuantía y solo en algunos tipos de cáncer. Por ejemplo, una de las últimas revisiones sobre la relación del cáncer con la afectividad negativa y la ira indica que el estilo de ira controlada y el afecto negativo no están asociados con el cáncer de mama, el melanoma o el riesgo global de cáncer, pero si es un factor de riesgo menor en el desarrollo del cáncer de próstata, colon-rectal y de pulmón. Por tanto, la asociación difiere en función del tipo de cáncer (White et al., 2007).

Estudios psiconeuroinmunológicos han analizado la influencia del estrés, la ansiedad y la depresión como factores de riesgo etiológicos en el crecimiento de la neoplasia no existiendo en la actualidad consenso acerca de su papel de variable desencadenante, pero si como variable moderadora que influye en la velocidad del proceso cancerígeno (Amigo et al., 2003; p. 200). Así, Shekelle et al. (1981) hallaron en su estudio que las personas que presentaban puntuaciones elevadas de depresión en un cuestionario de personalidad mostraban, diecisiete años más tarde, una probabilidad doble de haber muerto a causa de un cáncer. 
También la relación específica entre depresión y cáncer se ha estudiado desde diferentes perspectivas, encontrando multitud de estudios con resultados contradictorios. Por tanto, no se ha demostrado una asociación clara entre los factores psicológicos y el comienzo, exacerbación o la evolución de la enfermedad neoplásica, aunque estudios más recientes con mayor solidez metodológica han sugerido que la progresión del cáncer, y no su inicio, puede verse influida en mayor medida por factores psicosociales (cfr. Miralles et al., 2003). Para una revisión en mayor profundidad recomendamos al lector acudir a Rivero, Ramos, GarcíaLópez y Piqueras (2006).

\section{Infección por el virus de inmunodeficiencia humana (VIH)}

Los pacientes infectados por el VIH también presentan sintomatología ansiosa, como la excesiva preocupación, el miedo y la obsesión por ser portador del VIH o de presentar alguna de las llamadas enfermedades oportunistas, tales como el SIDA. La persona seropositiva está sometida a numerosos estresores que pueden ocasionar trastornos de adaptación, de ansiedad y depresión, entre otros. Los síntomas más predominantes son la ansiedad generalizada, la hipocondría, los comportamientos obsesivos, los pensamientos depresivos, la culpabilidad y el autocastigo (Tulldrá, Izquierdo, Fumaz y Ferrer, 2003). Las pruebas para detectar el VIH son un importante estresor. Se ha descrito una prevalencia alta de ansiedad y depresión en pacientes en periodos anteriores a la realización de las pruebas y descenso de dichos síntomas tras realizarlas. Así, el 25\% de los pacientes diagnosticados como "seropositivos" desarrollan tras las pruebas, trastornos de adaptación y trastornos mixtos ansioso-depresivos además de existir en ellos un riesgo de suicidio 36 veces superior a la población normal (Marzuk, 1988). Por su parte, Teva, Bermúdez, Hernández-Quero y BuelaCasal (2005) hallaron niveles de depresión, ansiedad e ira no expresada en pacientes con VIH, así como su asociación a los niveles de carga viral, grado de lipodistrofia y el estadio de infección.

Por último, la asociación entre emociones negativas y VIH-positivo ha sido también abordada al analizar la eficacia de las intervenciones cognitivoconductuales en la mejora de la salud mental y el funcionamiento inmunológico. Así las intervenciones cen- tradas en mejorar la sintomatología depresiva, ansiosa, de ira, estrés y el conteo de células CD4 han mostrado mejoras significativas en depresión, ansiedad, ira y estrés, si bien la evidencia es limitada para los efectos en el conteo de células CD4 (Crepaz et al., 2008).

\section{Trastornos cardiovasculares}

Varios estudios han mostrado que la depresión es un factor de riesgo significativo de enfermedad coronaria, infarto de miocardio y mortalidad cardiaca, así como también la ansiedad y el estrés (cfr. FernándezAbascal, Martín y Domínguez, 2003; pp. 100-101). Más concretamente, se ha relacionado la afectividad negativa como estado emocional de los pacientes con los trastornos cardiovasculares (Denollet, 1991). El concepto de afectividad negativa puede ser entendido como un estado emocional transitorio o como una diferencia persistente en el nivel general de afectividad. La afectividad negativa es un rasgo que refleja la tendencia a experimentar emociones negativas a través del tiempo y de situaciones (Watson y Clark, 1984). Este rasgo se solapa con el neuroticismo y la ansiedad rasgo, incluyendo sentimientos subjetivos de tensión, preocupación, ansiedad, ira y tristeza.

También existe una evidencia acumulada sobre la alta prevalencia de los trastornos psiquiátricos comórbidos con la enfermedad cardiovascular, concretamente con el episodio depresivo mayor (29\%), trastorno dístímico (15\%), trastorno depresivo mayor recurrente $(31 \%)$, trastorno de estrés postraumático (29\%) y trastorno de ansiedad generalizada (24\%) (Bankier, Januzzi y Littman, 2004).

Otro grupo de investigaciones han estudiado la asociación entre depresión y ansiedad con el pronóstico de pacientes cardíacos. Así, la depresión se ha relacionado con una peor evolución de los pacientes coronarios, ya que predispone a nuevos infartos y a la aparición de trastornos del ritmo cardíaco (Carinci et al., 1997). Respecto a la ansiedad, no sólo el trastorno de ansiedad generalizada sino también los trastornos fóbicos se han asociado al aumento de riesgo cardíaco en diversas poblaciones. En resumen, algunas investigaciones han encontrado que la depresión está asociada con alta mortalidad, en tanto en cuanto la ansiedad está asociada con baja mortalidad (Hermann, BrandDriehorst, Buss y Rüger, 2000), si bien no se ha docu- 
mentado una relación causal directa (Lespérance y Frasure-Smith, 2000).

Otro de los factores asociados a los trastornos cardiovasculares relacionado con la "afectividad negativa" ha sido el Complejo Hostilidad-Ira-Agresión o Síndrome Ira-Hostilidad-Agresión (AHI). Este síndrome ha recibido atención tanto como componente del Patrón de Conducta Tipo A como de forma aislada, siendo el que más se ha relacionado con la enfermedad cardiovascular, en especial el componente de Hostilidad. El complejo o síndrome AHI (Agresividad-Hostilidad-Ira) está formado por la ira, que es el componente emocional, la hostilidad, que hace referencia al componente cognitivo y la agresividad, que se refiere al aspecto conductual. Dicho síndrome está relacionado con trastornos psicofisiológicos, especialmente las alteraciones cardiovasculares (Fernández-Abascal et al., 2003).

Aunque el grado que alguien necesita para enojarse puede influir en el curso y el resultado de las enfermedades, la manera en que se expresa/maneja la ira tiene un impacto mucho mayor en el curso de la enfermedad y en el impacto. Al considerar como se expresa la ira, los investigadores han identificado tres estilos distintos de expresión de la ira: supresión de la ira, expresión de la ira y control de la ira (anger-in, anger-out $\mathrm{y}$ anger-control). Anger-in es cuando la persona suele afrontar la situación reprimiendo la expresión verbal o física, pero experimenta activación interna elevada, mientras que Anger-out implica manifestaciones de conducta airadas, verbales o físicas, hacia otras personas y/u objetos, pero sin intención de producir daño $(162,163)$. Por último el Control de la Ira (Anger-Control) es un estilo que se caracteriza porque la persona intenta canalizar su energía emocional proyectándola hacia fines más constructivos, para llegar a una resolución positiva del conflicto (Spielberger, Reheiser y Sydeman, 1995). Ser capaz de expresar ira parece disminuir el impacto negativo de ira en las funciones física y emocional. En cualquier caso, expresar ira puede tener consecuencias negativas tales como ser "socialmente inaceptable", crear conflictos y activar consecuencias negativas percibidas. Por tanto el estilo de Control de la ira parece el más adecuado. En este sentido, Krantz et al. (2006) hallaron que la relación entre factores psicosociales como son la ira y la hostilidad, los síntomas cardíacos y la enfermedad rrterial coronaria probada angiográficamente en mujeres consistía exactamente en que la alta Expresión de la Ira se asociaba a la presencia de EAC-Angiográfica, mientras que los rasgos de Ira/Hostilidad estaban asociados a un incremento de síntomas, sobre todo dolor torácico no asociado a angina en mujeres sin EAC-A.

Mención aparte merece la hipertensión arterial primaria o esencial por ser un factor de riesgo cardiovascular donde la ansiedad juega un papel importante. Es una alteración con etiología pluricausal donde uno o varios factores de riesgo están presentes para provocar una elevación de la presión arterial, siendo esos factores de riesgo principalmente conductuales. La Organización Mundial de la Salud ha reconocido el importante papel que puede jugar el estrés en la HTA, aunque también reconoce la dificultad de cuantificar esa influencia en el desarrollo de esta enfermedad (OMS, 1986). Entre los factores psicológicos que muestran mayor evidencia de correlación con la hipertensión, destacan la ansiedad y la ira, que desempeñan un importante papel en su desarrollo y mantenimiento, ya sea directamente por efectos sobre el sistema cardiovascular o indirectamente por su influencia en los factores conductuales, tales como el hábito alimenticio, el ejercicio físico, etc. (Amigo et al., 2003). Un perfil de sujeto hipertenso se caracteriza por un alto rasgo general de ansiedad, alta reactividad en los sistemas de respuesta cognitivo y fisiológico, y en menor medida en el sistema motor; y con reacciones de ansiedad intensas ante las situaciones de prueba o evaluación, las situaciones potencialmente fóbicas, las habituales en su vida diaria y, en menor medida, ante las situaciones interpersonales. En este sentido resulta de gran interés los resultados de una encuesta realizada por Patel (1984) en la que se preguntaba cuál era la causa más importante de la hipertensión tanto a población general como a afectados por la hipertensión. El 48 por 100 de los encuestados sin este trastorno y el 53 por 100 de los hipertensos encuestados atribuyeron a la categoría de tensión emocional, preocupaciones y ansiedad el papel causal más importante en la etiología de la enfermedad.

\section{Trastornos dermatológicos}

Desde el punto de vista fisiológico, la piel es uno de los órganos más sensibles a las emociones. Proba- 
blemente sea responsable de ello el origen embriológico común ectodérmico de la piel y del sistema nervioso que hace que ambas estructuras tengan en común multitud de neuromoduladores, péptidos vasoactivos y sistemas bioquímicos de información interna.

Los trastornos dermatológicos o cutáneos han sido considerados tradicionalmente como pertenecientes a la psicosomática, ya que se conoce que pueden ser precipitados o exacerbados por el estado emocional de la persona. En diferentes trastornos cutáneos, tales como la psoriasis, la dermatitis atípica, la urticaria, la alopecia areata, etc., se mantiene que existen muchos factores que pueden actuar como desencadenantes de los brotes o desempeñen un importante papel como factor mantenedor de la misma (Panconesi, 2000). Entre estos se hallan el estrés y las alteraciones emocionales como la ansiedad o la depresión, que pueden causarlos o exacerbarlos. También el acné se ha relacionado al estrés emocional, ya que es exacerbado mediante la liberación de determinadas hormonas como los glucocorticoides y andrógenos como consecuencia de la respuesta emocional a los estresores (Miralles et al., 2003).

Diversos estudios han sugerido también que los trastornos dermatológicos presentan una alta comorbilidad con trastornos ansiosos y afectivos. En principio esto estaría relacionado con la desfiguración facial o de otra parte del cuerpo que podría generar problemas psicológicos, tales como sentimientos de inferioridad, soledad y baja autoestima, así como retraimiento social. Sin embargo, solo una pequeña proporción de personas desarrolla trastornos psíquicos serios, como fobia social, ansiedad generalizada o depresión mayor (Folks y Kinney, 1992).

\section{Trastornos endocrinos}

Investigaciones recientes apoyan la existencia de una relación entre eventos vitales estresantes y el comienzo diabético. Debido a que estos estudios se han realizado con pequeñas poblaciones, deben interpretarse sus resultados con prudencia; sugieren que algunas personas son más vulnerables que otras a los efectos del estrés. El estrés crónico en una persona predispuesta a padecer diabetes (obesa y edad avanzada) puede constituir un elemento coadyuvante para desarrollar la enfermedad (Surwit et al., 2002). Así, los factores estresantes pueden precipitar su inicio menoscabando la regulación del metabolismo de los hidratos de carbono en el paciente, tanto asintomáticamente como produciendo hiperglucemias en la fase de estado.

Otros estudios han señalado que la presencia de síntomas depresivos incrementa el riesgo de desarrollar diabetes en un 22\% (Arroyo et al., 2004), mientras que otros como el estudio de Kessing, Nilsson, Siersma y Andersen (2003) no hallan esta relación, con lo cual tampoco se puede concluir nada al respecto.

Por último, algunos autores han señalado que los diabéticos presentan una mayor prevalencia de trastornos de ansiedad y depresión en contraste con la población no diabética (p.ej., Pierrot y Rubin, 1997).

En resumen, con independencia de otros factores parece existir un componente psicógeno que influye en la evolución de la enfermedad al condicionar la forma de adaptarse a los estresores sociales (el primero de los cuales es el propio diagnóstico de diabetes). En este componente psicógeno confluyen diferentes circunstancias, como son las primeras experiencias en relación con la diabetes, la respuesta de la familia de origen ante el diagnóstico, los rasgos de personalidad (neuroticismo, dramatismo, dependencia), el soporte sociofamiliar, el inicio temprano del proceso, las hospitalizaciones desde la adolescencia, las consecuencias familiares de la enfermedad, la responsabilidad que comporta la intervención activa del enfermo en su tratamiento y control, la certeza de cronicidad y de posibles complicaciones graves y la dificultad para mantener un control dietético. Todos estos factores pueden contribuir al desarrollo neurótico de la personalidad: hipocondría, tendencia a la inestabilidad emocional, irritabilidad, soledad, desesperanza y depresión.

\section{Trastornos gastrointestinales}

La función principal del sistema gastrointestinal es acomodar y guardar los alimentos ingeridos para, a continuación, molerlos, triturarlos y, finalmente, liberarlos de una manera coordinada a la luz intestinal. Dado que la función primordial del estómago es el vaciado gástrico, los problemas motores que se han relacionado con este fenómeno se pueden dar a cualquier nivel del aparato digestivo, tanto a nivel del esófago, como del estómago y/o de los intestinos. Por ello va- 
mos a describir qué papel juegan la ansiedad y la depresión en el funcionamiento de cualquiera de estos órganos.

En cuanto al esófago, algunos trabajos destacan una mayor comorbilidad entre trastornos afectivos, trastornos de ansiedad y trastornos de la motilidad esofágica (Handa et al., 1999). También existen datos a favor de que un trastorno psíquico ansioso o depresivo puede iniciar un trastorno esofágico y a su vez, la anormalidad en la motilidad del tracto puede incrementar la ansiedad.

En cuanto al funcionamiento del estómago, el estrés y los factores emocionales son considerados factores de riesgo en algunas enfermedades de la motricidad gástrica. Diversos estudios describen a un grupo de pacientes con manifestaciones típicas de gastroparesia o retraso de vaciado gástrico en los cuales no se halla causa orgánica identificable. Se trata de personas jóvenes, en quienes los factores psicológicos desempeñan un papel fundamental, por ejemplo en las pacientes con anorexia nerviosa o en los pacientes sometidos a situaciones de estrés. Determinadas condiciones clínicas como la infección por Helicobacter pylori, la dispepsia funcional no ulcerosa o el síndrome del colon irritable se han asociado en este grupo de pacientes con manifestaciones clínicas de gastroparesia (Overmier y Murison, 2000).

Respecto a la úlcera péptica, la teoría fisiopatológica más conocida es que el estrés produce una intensa secreción ácida e hipersecreción de peptina, mediada por la estimulación vagal, que acaba lesionando la mucosa, y ha sido considerada como paradigma de enfermedad psicosomática. Sin embargo, el descubrimiento del Helicobacter pylori y la evidencia de personas hiperestresadas sin lesión, así como la presencia de úlceras en personas sin psicopatología significativa han obligado a replantear la cuestión. Aún así, varios estudios recientes siguen corroborando que el estrés es un factor de riesgo independiente para el desarrollo y la recurrencia de la enfermedad ulcerosa. Concretamente, periodos transitorios de estrés se asocian a una mayor probabilidad de desarrollar ulceras de estomago que periodos de estrés largos y prolongados (e.g., Overmier y Murison, 2000). En la actualidad, aunque existe controversia acerca del papel de los factores psicológicos, lo que si parece claro es que la úlcera péptica debe ser considerada como un problema de salud de tipo biopsicosocial y a partir de ahí abrir un nuevo campo de estudio para esclarecer la relación entre los diferentes factores implicados (Tobón, Sandín y Vinaccia, 2005).

También se llegó a describir una personalidad "ulcerosa", persona con necesidades intensas de dependencia y deseos íntimos de ser cuidado y protegido, pero la evidencia actual rechaza este tipo de personalidad. En cambio, parece existir acuerdo en cuanto a que determinados factores psíquicos, independientes de la "personalidad ulcerosa", podrían influir tanto sobre estas conductas de riesgo como en la percepción y valoración de los síntomas ulcerosos por el paciente. Nos referimos a los hábitos de consumo (tabaco, alcohol, etc.), factores emocionales (personalidad con hostili$\mathrm{dad}$, ansiedad) y antecedentes familiares (cfr. Miralles et al., 2003).

Otros trabajos muestran una comorbilidad alta de la depresión mayor, los trastornos de pánico y agorafobia con la dispepsia no ulcerosa (Handa et al., 1999).

Por otra parte, parece contrastada la importancia de los factores psicológicos en la caracterización de los trastornos intestinales. Los datos de diferentes estudios indican que los individuos con síntomas gastrointestinales presentan mayor prevalencia de trastornos psíquicos que quienes están libres de estos síntomas (García-Vega, 2003). Dos de los trastornos más estudiados han sido la enfermedad inflamatoria intestinal (colitis ulcerosa y enfermedad de Crohn) y el síndrome de intestino irritable.

Existen datos a favor de la influencia de las emociones y los hábitos conductuales en la fisiología gastrointestinal, más concretamente en la enfermedad inflamatoria intestinal (Anton, 1999). Parece demostrada una clara relación entre el estrés emocional y la exacerbación del proceso inflamatorio, así como con la percepción de las molestias sintomáticas. Incluso se han descrito rasgos de personalidad característicos, obsesivo-compulsivos y alexitímicos. Concretamente, se ha hallado una elevada incidencia de trastornos depresivos y ansiosos entre los pacientes con enfermedad inflamatoria intestinal (García-Vega, 2003). 
Respecto al síndrome del intestino irritable es una de las enfermedades más frecuentes del aparato digestivo. Las hipótesis sobre su etiología son variadas aunque se sabe que los factores psicológicos tienen un papel importante. Según algunos de los trabajos publicados, hasta el $70 \%$ de los pacientes cumple criterios para algún trastorno psiquiátrico, siendo los trastornos de ansiedad y los depresivos los más frecuentes, mientras que otros encontraron en su investigación que, en un notable porcentaje, los trastornos de ansiedad ya se encontraban presentes antes de la aparición de los síntomas gastrointestinales (cfr. Fernández, 2003).

Por tanto, los aspectos emocionales que se han relacionado con los trastornos gastro-intestinales funcionales (combinaciones de síntomas gastro-intestinales que no se explican por alteraciones bioquímicas o estructurales y que no muestran anormalidades en estudios médicos) son muy numerosos. Así, se ha hallado que la tendencia agresiva y la hipocondría pueden incidir en la enfermedad gastrica, que la ansiedad y la depresión aumentan el dolor abdominal, la afectividad negativa (tendencia a experimentar ira, disgusto, asco, culpa, temor o depresión) se relaciona con las quejas físicas, etc. Igualmente se considera que las personas con enfermedades gastrointestinales suelen tener niveles más elevados de miedo, enojo, ansiedad y tristeza, así como ansiedad rasgo y neuroticismo, irritabilidad $u$ hostilidad, etc. y viceversa, es decir, aquellas personas que se han pasado por una elevada frustración relacionada con sus metas y tiene reacciones de agresividad, o están afectadas en su estado de ánimo, fatigados, con sentimientos de culpa, con poco interés por las cosas, tensionadas o preocupadas excesivamente, inquietas o irritables son más propensas a padecer enfermedades gastrointestinales (Juárez, Cano y Olarte, 2004).

\section{Enfermedades neurológicas}

Las enfermedades neurológicas son las que presentan una mayor coexistencia con la depresión y la ansiedad (e.g., Robertson, 1997).

El dolor de cabeza es el síntoma neurológico más frecuente y uno de los motivos de consulta más frecuentes en general (se estima que entre un 10\%-20\% de los casos es el síntoma principal). Existen diferentes tipos: cefalea tensional, migraña, cefalea en racimos y dolores de cabeza diversos. Tanto la ansiedad como la depresión son comunes en los dolores de cabeza crónicos (Maizels, 2004).

Respecto a la cefalea tensional, muchas personas son susceptibles a los dolores de cabeza en épocas de mayor estrés emocional y puede constituir un síntoma importante en síndromes ansiosos y depresivos (Maizels, 2004). La explicación a estas cefaleas es que el exceso de ansiedad suele producir contracción de la musculatura de la cabeza y el cuello. Si ésta se prolonga varias horas se produce constricción vascular e isquemia, que sería la causante del dolor. Las personas caracterizadas por patrones de personalidad del tipo A son especialmente proclives a este trastorno. Se ha estimado que hasta el $95 \%$ de estos pacientes padecen síntomas de ansiedad y/o depresión, bien de naturaleza reactiva o endógena. Además algunos estudios indican que el padecimiento de cefaleas prolongadas puede desencadenar trastornos psicológicos que, a su vez, pueden convertirse en variables mantenedoras del trastorno psicológico y potenciando la gravedad de la cefalea en futuros ataques. Concretamente, algunos estudios epidemiológicos indican que estos enfermos se caracterizan por rasgos de ansiedad, neuroticismo y depresión anormalmente altos (Stewart, Shechter y Rasmussen, 1994). En cuanto a la relación temporal, según algunos estudios la depresión se inicia después de la cefalea, existiendo una comorbilidad superior al 20\% (Merikangas, Angst e Isler, 1990).

El estrés y los trastornos de ansiedad también son un precipitante de la migraña. Según los estudios epidemiológicos, los trastornos de ansiedad preceden a la migraña, aunque probablemente interaccionan con otros precipitantes que aumentan su vulnerabilidad, pero sin desencadenarla necesariamente. Así pues, el estrés puede iniciar directamente la migraña en pacientes biológicamente propensos y puede potenciar indirectamente o intensificar el desencadenamiento de la misma. Posteriormente, las migrañas presentan mayor riesgo de asociación a crisis de pánico o ansiedad.

Existen otras enfermedades neurológicas más graves que también presentan una comorbilidad alta con el trastorno depresivo mayor así como alto riesgo de suicidio en los 5 años siguientes a la lesión, especialmente en pacientes con epilepsia, esclerosis múltiple o lesiones de la medula espinal. También se ha documen- 
tado una incidencia en torno al $40 \%$ de depresión asociada a accidentes vasculares cerebrales agudos (Starkstein, Fedoroff, Price, Leiguarda y Robinson, 1993).

Otra enfermedad muy estudiada es la demencia. Algunos estudios consideran que esta enfermedad neurológica desencadena trastornos depresivos (Ballard, Bannister, Solis, Oyebode y Wilcock, 1993). Otra enfermedad neurológica como el Parkinson, también presenta altos niveles de psicopatología asociada, tanto ansiedad como depresión. Sin embargo, algunos autores recuerdan que hay que ser cuidadoso con esta asociación Parkinson-depresión, ya que el estado de ánimo del paciente puede deberse a estados transitorios de disforia más propios de las exacerbaciones de la enfermedad neurológica (Richard, 2005).

Por último, se ha hallado una asociación entre la ansiedad y la depresión y la esclerosis múltiple. Así, hasta el 75\% de personas con esta enfermedad lleva asociado problemas psicológicos y psiquiátricos (trastorno de pánico, trastorno depresivo, trastorno bipolar, etc.). Además, la depresión y la ansiedad influyen en la relación entre la Esclerosis Múltiple y la calidad de vida, ya que los individuos con más síntomas de ansiedad y depresión presentan mayor incapacidad física y peor calidad de vida (Janssens et al., 2004).

\section{Trastornos respiratorios}

Actualmente no se ha podido demostrar la influencia etiopatogénica en la enfermedad respiratoria de los factores psicológicos, pero sí se acepta la posible influencia de los factores psicosociales sobre la evolución del asma bronquial o en el mantenimiento de algunos de los síntomas. Así, existen múltiples estudios metodológicamente rigurosos que demuestran el importante papel que la ansiedad asociada a las crisis de asma desempeña sobre la evolución del asma bronquial y de la enfermedad respiratoria en general (Bosley, Corden y Cochrane, 1996). La ansiedad de algunos pacientes se asocia a una peor evolución de la enfermedad, al aumentar la sintomatología y transmitir una mayor sensación de gravedad, lo que lleva a tratamientos más urgentes, frecuentes, agresivos y costosos. La depresión también puede influir negativamente sobre la evolución del paciente asmático, conduciendo a un autocuidado pobre, con incumplimiento de las prescrip- ciones médicas y tendencia a la indiferencia ante los síntomas respiratorios (Vázquez, Romero-Frais y Sández, 2003).

\section{Trastornos reumatológicos}

Muchas enfermedades reumatológicas cursan de forma crónica, produciendo dolor, deformación e incapacidad funcional. Repercuten directamente sobre la calidad de vida del paciente, afectando su actividad laboral, social y familiar. Con frecuencia los síntomas y signos de la alteración músculo-esquelética se solapan con la sintomatología propia de una afectación emocional (EPISER, 2001).

En una enfermedad como la artritis reumatoide, el dolor y el deterioro físico pueden causar distintos síndromes psiquiátricos, de los que el más estudiado es el depresivo. Se estima la prevalencia de depresión mayor en torno al $17 \%$ y la de distimia en torno al $40 \%$ (Miralles et al., 2003). Estos factores emocionales han sido implicados en la evolución de la artritis reumatoide de forma desfavorable, en el sentido de poca motivación, depresión no asociada al dolor y mal control de impulsos.

En pacientes con dolor crónico se considera que la presencia de síntomas de ansiedad es muy significativa y tanto la ansiedad como la depresión es un factor facilitador de la percepción del dolor (Melzack y Wall, 1965).

En cuanto a la fibromialgia, existe unanimidad en la literatura con relación a la presencia de alteraciones emocionales en estos pacientes (Rice y Pisetsky, 1999). Se puede decir que síntomas como tristeza, ansiedad, fatiga, insomnio, irritabilidad, falta de concentración, desinterés, apatía e hipocondría forman parte del cuadro clínico. Es frecuente la concomitancia de la fibromialgia con otras enfermedades médicas con acreditado contenido psicológico, como el síndrome del intestino irritable, la migraña, problemas de sensibilización alérgica y en especial el síndrome de fatiga crónica.

\section{CONCLUSIONES}

En este artículo se ha intentado mostrar una síntesis de los datos relativos a la influencia de los factores 
emocionales, específicamente las emociones negativas clásicas (tristeza-depresión, miedo-ansiedad, ira y asco) en el proceso de salud-enfermedad. Estos factores se han asociado con las enfermedades mentales y físicas como variables influyentes en el inicio, desarrollo y mantenimiento. Se han hipotetizado básicamente dos vías explicativas generales. La primera hace referencia a la influencia de las emociones negativas en la conducta, de manera que interfieren en los hábitos saludables y fomentan el desarrollo de conductas inadecuadas que ponen en peligro nuestra salud. El segundo mecanismo se refiere a la repercusión de la activación psicofisiológica en los sistemas orgánicos, afectando a la inmunidad, por ejemplo. Por último, se han descrito algunos de los vínculos entre las emociones negativas y los trastornos mentales y físicos. Así, no solo existe una influencia de las emociones negativas en el inicio y el curso de estos trastornos, sino que también se reconoce una alta comorbilidad tanto de los trastornos mentales como las enfermedades médicas con los trastornos ansiosos y depresivos, y con la ira y el asco patológico, frecuentemente como consecuencia del padecimiento de las mismas.

\section{REFERENCIAS}

Alonso, C. (2006). Repercusión psicológica de la enfermedad en el paciente oncológico, adaptación e intervención. En Interpsiquis, 2006. [En línea]. Recuperado el 7 de febrero de 2006. Disponible en Internet <URL:http://www.psiquiatria.com>

American Psychiatric Association (2000). Diagnostic and Statistical Manual of Mental Disorders (fourth edition, text revision). Washington, DC: APA.

Amigo, I., Fernandez, C. y Perez, M. (2003). Manual de Psicología de la Salud (2 ${ }^{\mathrm{a}}$ ed.). Madrid: Pirámide.

Anton, P.A. (1999). Stress and mind-body impact on the course of inflammatory bowel diseases. Seminars in Gastrointestinal Disease, 10, 14-19.

Ballard, C., Bannister, C., Solis, M., Oyebode, F. y Wilcock, G. (1996). The prevalence, associations and symptoms of depression amongst dementia sufferes. Journal of Affective Disorders, 36, 135144.

Bankier, B., Januzzi, J.L. y Littman, A.B. (2004). The high prevalence of multiple psychiatric disorders in stable outpatients with coronary heart disease. Psychosomatic Medicine, 66, 645-650.

Barlow, D. H. (2002). Anxiety and its disorders: The nature and treatment of anxiety and panic (2nd ed.). New York: Guilford Press.

Beck, A.T., Rush, A.J., Shaw, B.F. y Emery, G. (1983). Terapia cognitiva de la depresión. Bilbao: Desclée de Brouwer.

Becoña, E. Vázquez, F. y Oblitas, L. (2004). Promoción de los estilos de vida saludables. En Investigación en Detalle Número 5. [En línea]. ALAPSA. Bogotá. Consultado el 7 de Febrero de 2006. Disponible en Internet <URL:http:// www.alapsa.org/detalle/05/index.html>

Bosley, C.M., Corden, M. y Cochrane, G.M. (1996). Psychosocial factors and asthma. Respiratory Medicine, 90, 453-457.

Cano-Vindel, A. (2004). Costes de los trastornos de ansiedad. En Información General sobre la Ansiedad y el Estrés: Los Trastornos de Ansiedad (Sociedad Española para el Estudio de la Ansiedad y el Estrés). Recuperado de: http://www.ucm.es/ info/seas/ta/costes/index.htm

Cano-Vindel, A. y Miguel-Tobal, J. J. (2001). Emociones y Salud. Ansiedad y Estrés, 7, 111-121.

Carey, C.L. y Harris, L.M. (2005). The origins of bloodinjection fear/phobia in cancer patients undergoing intravenous chemotherapy. Behaviour Change, $22,212-219$.

Carinci, F., Nicolucci, A., Ciampi, A., Labbrozzi, D., Bettinardi, O., Zotti, A. M. y Tognoni, G. (1997). Role of interactions between psychological and clinical factors in determining 6-month mortality among patients with acute myocardial infarction. European Hearth Journal, 18, 835-845.

Chóliz, M. (2005). Psicología de la Emoción: el proceso emocional. España. Departamento de Psicología Básica. Universidad de Valencia. Pág. 1-33. Recuperado el 20 de setiembre de 2005 en: www.uv.es/=choliz 
Crepaz, N., Passin, W.F., Herbst, J.H., RAma, S.M., Malow, R.M., Purcell, D.W. y Wolitski, R.J. (2008). Meta-analysis of cognitive-behavioral interventions on HIV-positive persons' mental health and immune functioning. Health Psychology, 27, 4-14.

Eckhardt, C., Jamison, T. R. y Watts, K. (2002). Anger experience and expression among male dating violence perpetrators during anger arousal. Journal of Interpersonal Violence, 17, 1102-1114.

Ellis, A. y Grieger, R. (1981). Manual de terapia racional-emotiva. Bilbao: Desclée de Brouwer (Trabajo original publicado en 1977).

Ellis, A. y Grieger, R. (1990). Manual de terapia racional-emotiva: Vol. II. Bilbao: Desclée de Brouwer (Trabajo original publicado en 1985).

Engen, R., Levy, N. y Schlosberg, H. (1958). The dimensional analysis of a new series of facial expressions. Journal of Expermiental Psychology, 55, 454-458.

EPISER (2001). Prevalencia e impacto de las enfermedades reumáticas en la población adulta española. SER 2001. Meck Sharp \& Dohme de España (www.msd.es).

Fernández, C. (2003). Guía de tratamientos psicológicos eficaces en el síndrome del intestino irritable. En M. Pérez, J.R. Fernández, C. Fernández e I. Amigo (Coord.), Guía de tratamientos psicológicos eficaces II. Psicología de la Salud (pp.169-185). Madrid: Pirámide.

Fernández-Abascal, E.G. y Palmero, F. (1999). Emociones y salud. Barcelona: Ariel.

Fernández-Abascal, E.G., Marín, M.D. y Domínguez, F.J. (2003). Guía de tratamientos psicológicos eficaces en los trastornos cardiovasculares. En M. Pérez, J.R. Fernández, C. Fernández e I. Amigo (Coord.), Guía de tratamientos psicológicos eficaces II. Psicología de la Salud (pp. 93-121). Madrid: Pirámide.

Folks, D.G. y Kinney, F.C. (1992). The role of psychological factors in dermatologic conditions. Psychosomatics, 33, 45-54.

Friedman, E. S. y Thase, M. E. (1995). Trastornos del estado de ánimo. En: V. E. Caballo, G. Buela-Casal y Carrobles, J. A. (dirs.). Manual de Psicopatología y Trastornos Psiquiátricos. Vol. 1. Madrid: Siglo XXI.

García-Vega, E. (2003). Guía de tratamientos psicológicos eficaces en la enfermedad inflamatoria intestinal. En: M. Pérez, J.R. Fernández, C. Fernández e I. Amigo (Coord.), Guía de tratamientos psicológicos eficaces II. Psicología de la Salud (pp. 188-197). Madrid: Pirámide.

Gorenstein, E.E., Tager, F.A., Shapiro, P.A., Monk, C. y Sloan, R.P. (2007). Cognitive-Behavior Therapy for Reduction of Persistent Anger. Cognitive and Behavioral Practice, 41, 168-184.

Haidt, J., McCauley, C.R. y Rozin, P. (1994). A scale to measure disgust sensitivity. Personality and Individual Differences, 16, 701-713.

Handa M, Mine K, Yamamoto H, Tsutsui S, Hayashi H, Kinukawa N. y Kubo C. (1999). Esophageal motility and psychiatric factors in functional dyspepsia patients with or without pain. Digestive Diseases and Sciences, 44, 2094-2098.

Hermann, C., Brand-Driehorst, S., Buss, U. y Rüger, U. (2000). Effects of anxiety and depression on 5year mortality in 5057 patients referred for exercise testing. Journal of Psychosomatic Research, 48, 455-462.

Izard, C. E. (1993). Organizacional and motivacional functions of discrete emotions. En: M. Lewis (Ed.), Handbook of emotions (pp. 631-641). Nueva York: Guilford Press.

Janssens, A.C., van Doorn, P.A., de Boer, J.B., van der Meche, F.G., Passchier, J. y Hintzen, R.Q. (2004). Perception of prognostic risk in patients with multiple sclerosis: the relationship with anxiety, depression, and disease-related distress. Journal of Clinical Epidemiology, 57, 180-186

Juárez, F., Cano, M.A. y Olarte, M. (2004). Factores psicológicos asociados a síntomas dispépticos. Psicología y Salud, 14, 43-55.

Kessing, L.V., Nilsson, F.M., Siersma, V. y Andersen, P.K. (2003). Increased risk of developing diabetes in depressive and bipolar disorders?. Journal of Psychiatric Research, 38, 395-402.

Kop, W.J., Gottdiener, J.S., Tangen, C.M., Fried, L.P., 
McBurnie, M.A., Walston, J., Newman, A., Hirsch, C. y Tracy, R.P. (2002). Inflammation and coagulation factor in persons $>65$ years of age with symptoms of depression but without evidence of myocardial ischemia. American Journal of Cardiology, 89, 419-424.

Krantz, D.S., Olson, M.B., Francis, J.L., Phankao, C., Bairey, C.N., Sopko, G., Vido, D.A., Shaw, L.J., Sheps, D.S., Pepine, C.J. y Matthews, K.A. (2006). Anger, Hostility, and Cardiac Symptoms in Women with Suspected Coronary Artery Disease: The Women's Ischemia Syndrome Evaluation (WISE) Study. Journal of Women's Health, 15, 1214-1223.

Lazarus, R. S. (1991). Emotion and adaptation. New York: Oxford University Press.

Lazarus, R.S. (1993). Coping Theory and research: Past, present and future. Psychosomatic Medicine, 55, 234-247.

Lespérance, F. y Frasure-Smith, N. (2000). Depression in patients with cardiac disease: a practical review. Journal of Psichosomatic Research, 48, 379-391.

Leventhal, H., Prochaska, T.R. y Hirschman, R.S. (1985). Preventive health behavior across the lifespan. En: J.C. Rosen y L.J. Solomon (Eds.), Prevention in health psychology. Hanover, NH: University Press of New England.

Lewinsohn, P.M, Gotlib, I.H y Hautzinger, M. (1997). Tratamiento conductual de la depresión. En V.E. Caballo, Manual para el tratamiento cognitivoconductual de los trastornos psicológicos. Madrid: Siglo XXI.

Lundeberg, K., Stith, S. M., Penn, C. E. y Ward, D. B. (2004). A comparison of nonviolent, psychologically violent, and physically violent male college daters. Journal of Interpersonal Violence, 19, 11911200.

Maizels, M. (2004). The patient with daily headaches. American Family Physician, 70, 2299-2306.

Martín, M. (2005). PSICRON: Una metodología computarizada para optimizar la asistencia, la investigación y la docencia con pacientes con enfermedades crónicas. En: P.E. Vera-Villaroel y L.A. Oblitas (Eds.), Manual de Escalas y Cuestionarios Iberoamericanos en Psicología
Clínica y de la Salud. Bogotá: PSICOM (www.psicologiacientifica.com).

Marzuk, P.M., Tierney, H., Tardiff, K., Gross, E.M., Morgan, E.B., Hsu, M.-A. y Mann, J.J. (1988). Increased risk of suicide in persons with AIDS. Journal of the American Medical Association, 259, 1333-1337.

Melzack, R. y Wall, P.D. (1965). Pain mechanisms: A new theory. Science, 150, 971-979.

Miguel-Tobal, J.J.; Casado, M.I.; Cano-Vindel, A. y Spielberger, C.D. (1997). El estudio de la ira en los trastornos cardiovasculares mediante el empleo del Inventario de Expresión de Ira Estado-Rasgo STAXI. Ansiedad y Estrés, 3, 5-20.

Miralles, L., Otin, R y Rojo, J.E. (2003). Factores psicológicos que afectan al estado físico. Medicine, 8, 5654-5664.

Nezu, A.M., Nezu, C.M. y Blissett, S.E. (1988). Sense of humor as a moderator of the relation between stressful events and psychological distress: A prospective analysis. Journal of Personality and Social Psychology, 54, 520-525.

Nicholson, R.A., Houle, T.T., Rhudy, J.L y Norton, P.J. (2007). Psychological Risk Factors in Headache. Headache, 47, 413-426.

Norlander, B. y Eckhardt, C. (2005). Anger, hostility, and male perpetrators of intimate partner violence: A meta-analytic review. Clinical Psychology Review, 25, 119-152.

Oatley, K. (1992). Best laid schemes: The psychology of emotions. New York: Cambridge University Press.

Oblitas, L. (2004). Manual de psicología clínica y de la salud hospitalaria. Bogotá: PSICOM (www.psicologiacientifica.com).

Oblitas, L. y Becoña, E. (2000). Psicología de la Salud: antecedentes, desarrollo, estado actual y perspectivas. En L. Oblitas, y E. Becoña (Eds.), Psicología de la salud (pp. 11-52). México: Plaza y Valdés.

Organización Mundial de la Salud (1986). Prevención y lucha contra las enfermedades cardiovasculares en la comunidad. Ginebra: OMS. 
Organización Mundial de la Salud (1992). Clasificación Internacional de Enfermedades (décima edición). Ginebra: OMS.

Overmier, J. B. y Murison, R. (2000). Anxiety and helplessness in the face of stress predisposes, precipitates, and sustains gastric ulceration. Behavioural Brain Research, 110, 161-174.

Panconesi E. (2000). Psychosomatic dermatology: past and future. International Journal of Dermatology, 39, 732-734.

Pierrot, M. y Rubin, R. (1997). Levels and risks of depression and anxiety symptomatology among diabetic adults. Diabetes Care, 20, 585-590.

Piqueras, J. A., Martínez, A., Ramos, V., Rivero, R. y García-López, L.J. (2006). Ansiedad, depresión y salud. En L. A. Oblitas (Ed.), Psicología de la Salud y Enfermedades Crónicas. Bogotá: PSICOM Editores (www.psicomeditores.com).

Plutchik, R. (1980). Emotion: a psychoevolutionary synthesis. New York: Harper and Row. I

Ramos, V., Rivero, R. Piqueras, J. A. y García-López, L.J. (2006). Psiconeuroinmunología. En: L. A. Oblitas (Ed.), Psicología de la Salud y Enfermedades Crónicas. Bogotá: PSICOM Editores (www.psicomeditores.com).

Rice, J.R. y Pisetsky, D.S. (1999). Pain in the Rheumatic Diseases. Practical aspects of diagnosis and treatment. Rheumatic Disease Clinics of North America, 25, 15-30.

Richard, I.H. (2005). Anxiety disorders in Parkinson's disease. Advances in Neurology, 96, 42-55.

Rivero, R., Ramos, V., García-López, L.J. y Piqueras, J. A. (2006). Psicooncología. En: L. A. Oblitas (Ed.), Psicología de la Salud y Enfermedades Crónicas. Bogotá: PSICOM Editores (www.psicomeditores.com).

Robertson, M.M. (1997). Depresión en los trastornos neurológicos. En: M.M. Robertson y C.L.E. Katona (Eds.), Depresión y enfermedades somáticas. Perspectivas en psiquiatría. Volumen 6. Chichester: John Wiley \& Sons Ltd.

Rozin, P., Haidt, J. y McCauley, C.R. (2000). Disgust. In M. Lewis y J.M. Haviland-Jones (Eds.), Hand- book of emotions, 2nd Edition (pp. 637-653). New York: Guilford Press.

Sandín, B. y Chorot, P. (1995). Concepto y categorización de los trastornos de ansiedad. En: A. Belloch, B. Sandín y F. Ramos (Eds.), Manual de Psicopatología: Vol. 2 (pp. 53-80). Madrid: McGraw-Hill.

Sawchuk, C. N., Lohr, J. M., Tolin, D. F., Lee, T. C., \& Kleinknecht, R. A. (2000). Disgust sensitivity and contamination fears in spider and blood-injectioninjury phobias. Behaviour Research and Therapy, 38, 753-762.

Shekelle, R.B., Raynor, W.J.Jr., Osfeld, A.M., Garron, D.C., Bieliauskas, L.A., Liu. S.C. Malis, C. y Paul, O. (1981). Psychological depression and 17 years risk of death from cancer. Psychosomatic Medicine, 43, 117-125.

Spencer, H. (1890). The principles of psychology (Vol. 1). New York: Appleton.

Spielberger, C., Reheiser, E. y Sydeman, S. (1995). Measuring the experience, expression, and control of anger. In H. Kassinove (ed.), Anger Disorders: Definition, Diagnosis, and Treatment (pp. 49-68). Washington, DC: Taylor \& Francis.

Starkstein, S.E., Fedoroff, J.P., Price, T.R., Leiguarda, R. y Robinson, R.G. (1993). Apathy following cerebrovascular lesions. Stroke, 24, 1625-1630.

Stewart, W.F., Shechter, A. y Rasmussen, B.K. (1994). Migraine prevalence. A review of population based studies. Neurology, 44, 17-23.

Suls, J., \& Bunde, J. (2005). Anger, anxiety, and depression as risk factors for cardiovascular disease: The problems and implications of overlapping affective dispositions. Psychological Bulletin, 131, 260-300.

Teva, I., Bermúdez, M.P., Hernández, J. y Buela-Casal, G. (2005). Evaluación de la depresión, ansiedad e ira en pacientes con VIH/SIDA. Salud Mental, 38, 40-49.

Tobón, S., Sandín, B. y Vinaccia, S. (2005). La úlcera péptica: ¿un trastorno gastrointestinal infeccionso o biopsicosocial?. Revista de Psicopatología y Psicología Clínica, 10, 95-100. 
Tolin, D.F., Woods, C.M. y Abramowitz, J.S. (2006). Disgust sensitivity ana obsessive-compulsive symptoms in a non-clinical sample. Journal of Behavior Therapy and Experimental Psychiatry, 37, 30-40.

Tulldrá, A., Izquierdo, I., Fumaz, C.R. y Ferrer, M.J. (2003). Guía de tratamientos psicológicos eficaces en la infección por el virus de inmunodeficiencia humana. En: M. Pérez, J.R. Fernández, C. Fernández e I. Amigo (Coord.), Guía de tratamientos psicológicos eficaces II. Psicología de la Salud (pp. 199-213). Madrid: Pirámide.

Vázquez, C. (1990). Trastornos del estado de ánimo: aspectos clínicos. En: F. Fuentenebro y C. Vázquez (eds.), Psicología Médica, Psicopatología y Psiquiatría (Vol. II). Madrid: McGraw-Hill Interamericana.

Vázquez, M. I., Romero-Frais, E. y Sández, E. (2003). Guía de tratamientos psicológicos eficaces en el asma bronquial. En: M. Pérez, J.R. Fernández, C. Fernández e I. Amigo (Coord.), Guía de tratamientos psicológicos eficaces II. Psicología de la Salud (pp. 23-55). Madrid: Pirámide.

Villagrán, J. M. (1996). La depresión. Madrid: Aguilar.
Watson, D., \& Clark, L. A. (1984). Negative affectivity: The disposition to experience aversive emotional states. Psychological Bulletin, 96, 465-490.

White, V.M., English, D.R., Coates, H., Lagerlund, M., Borland, R. y Giles, G.G. (2007). Is cancer risk associated with anger control and negative affect? Findings from a prospective cohort study. Psychosomatic Medicine, 69, 667-74.

Whybrow, P.C., Akiskal, H.S. y McKinney, W.T. (1984). Mood disorder: Toward a new psychobiology. Nueva York: Plenum.

Woodworth, R.S. (1938). Experimental Psychology. New York: Henry Holt.

Wundt, W. (1896). Grundriss der psychologie. Engelmann, Stuttgart, Germany.

Yerkes, R.M. y Dodson, J.D. (1908). The Relation of Strength of Stimulus to Rapidity of Habit-Formation. Journal of Comparative Neurology and Psychology, 18, 459-482.

Zajonc, R. B. (1980). Feeling and thinking: Preferences need no inferences. American Psychologist, 35, 151-175.

Fecha de recepción: 15 de enero, 2010

Fecha de aceptación: 18 de febrero, 2010 